\title{
Algorithmic Trading with Learning
}

\author{
Álvaro Cartea $^{\mathrm{a}}$, Sebastian Jaimungal ${ }^{\mathrm{b}}$, Damir Kinzebulatov ${ }^{\mathrm{c}}$ \\ ${ }^{a}$ Department of Mathematics, University of Oxford, Oxford, UK \\ ${ }^{b}$ Department of Statistical Sciences, University of Toronto, Toronto, Canada \\ ${ }^{c}$ The Fields Institute for Mathematical Sciences, Toronto, Canada
}

\begin{abstract}
We propose a model where an algorithmic trader takes a view on the distribution of prices at a future date and then decides how to trade in the direction of her predictions using the optimal mix of market and limit orders. As time goes by, the trader learns from changes in prices and updates her predictions to tweak her strategy. Compared to a trader who cannot learn from market dynamics or form a view of the market, the algorithmic trader's profits are higher and more certain. Even though the trader executes a strategy based on a directional view, the sources of profits are both from making the spread as well as capital appreciation of inventories. Higher volatility of prices considerably impairs the trader's ability to learn from price innovations, but this adverse effect can be circumvented by learning from a collection of assets that co-move. Finally, we provide a proof of convergence of the numerical scheme to the viscosity solution of the dynamic programming equations which uses new results for systems of PDEs.

Keywords: Algorithmic Trading; High Frequency Trading; Nonlinear Filtration; Brownian Bridge; Stochastic Optimal Control; Adverse Selection
\end{abstract}

\section{Introduction}

Information is a valuable asset, but being better informed than others is money. Modern trading algorithms employ unprecedented computer power to process information and make trading decisions. In this paper we show how an informed trader (IT) who has conceived a view of the market encoded in a prior on price changes, devises a strategy to trade in a single stock or group of stocks to maximize expected profits. The strategy is rooted in the IT's superior

Email addresses: alvaro.cartea@maths.ox.ac.uk (Álvaro Cartea), sebastian.jaimungal@utoronto.ca (Sebastian Jaimungal), dkinzebu@fields.utoronto.ca (Damir Kinzebulatov)

SJ thanks NSERC and GRI for partially funding this research. This paper has benefited from comments of seminar participants at the Isaac Newton Institute, University College London, Rutgers University. For comments and suggestions on earlier drafts, we are thankful to Ryan Donnelly, Jason Ricci, Chris Rogers. 
ability to constantly update her predictions of future prices and her ability to decide when to use limit and market orders to trade in and out of positions.

Trading aggressively with market orders is expensive, but guarantees execution, whereas a more passive and cheaper alternative is limit orders, but the time of execution is uncertain. The choice of order type depends on many factors including: accumulated inventory, remaining time before position has to be closed, and more importantly, how good are the price predictions. As time goes by, the IT updates her prior with new information and the strategy becomes more aggressive to accumulate or unwind inventories as the IT becomes more confident about the future direction of prices. For example, if the IT's initial prior is that the price of a particular stock will increase between now and a future date, then one expects the IT to initially accumulate a long position and then unwind it nearer the end of the strategy. The key question is how to do this optimally by choosing the right mix of market and limit orders at both ends of the strategy, taking into account the uncertainty in her predictions, as well as how to ride price changes and inventory exposure in between.

Most of the effort in the mathematical literature on algorithmic trading has focused on two classical problems: (i) optimal liquidation/acquisition (see, e.g., Almgren (2003), Obizhaeva and Wang (2013), Kharroubi and Pham (2010), Bayraktar and Ludkovski (2011), Bayraktar and Ludkovski (2014), Gatheral et al. (2012), Schied (2013), Guéant and Lehalle (2015)), and (ii) market making (see, e.g., Ho and Stoll (1981), Avellaneda and Stoikov (2008), Cartea et al. (2014), Cartea and Jaimungal (2015b), Guéant et al. (2012), Cartea and Jaimungal (2013)). This paper contributes to the growing literature in many aspects and in a new direction. It is the first paper, to the authors' knowledge, that incorporates information and dynamic learning in an algorithmic trading problem by using stochastic control methods. The trader learns from a collection of assets (i.e. uses the current prices to update the prior) and trades in all or a subset of them executing directional strategies. Moreover, we show how trading is done using both limit and market orders, which is something that few papers take into account - exceptions are Guilbaud and Pham (2013), Cartea and Jaimungal (2015a), and Cartea et al. (2015).

Our results show how the IT learns from changes in prices to tweak her strategy and maximize gains from trading. Although the strategy is devised to profit from a directional view of prices, the profits stem from both making the spread as well as capital appreciation of inventories. However, a key variable that influences the efficacy of learning, and hence the profitability of the strategy, is the volatility of prices. For example, we use simulations to show that if the IT is only trading in one asset and only learning from the dynamics of the same asset, then the higher the volatility, the more difficult it becomes to extract information from innovations in prices and the strategy turns much more conservative: it executes fewer market orders and avoids holding large inventory positions in the asset. In extreme cases where volatility is too high, the IT's ability to learn from price dynamics is impaired and she cannot 
execute a strategy based on directional price predictions. Thus, her strategy behaves like that of a market maker who wishes to bear very little inventory risk - passive orders are sent in such a way to ensure that inventories never stray too far away from zero.

The simulations also show how the IT's strategy compares to that of others who trade with lesser quality information or who are unable to learn from market dynamics. The IT's strategy is superior in both (higher) mean and (lower) standard deviation of profits; the IT's strategy second-order stochastically dominates the other traders' strategies. Furthermore, we also show that the profitability of trading in assets that exhibit higher volatility is considerably improved if the IT is able to learn from other of assets that co-move.

To solve for the optimal strategy used by the IT we adopt the dynamic programming principle to derive a quasi-variational inequality (QVI) which we solve numerically. We prove that the numerical scheme we employ does indeed converge to the unique viscosity solution of the QVI. Since inventories are discrete we cannot directly apply classical results to prove convergence of the scheme so we construct the proof from first principles. In doing so we use the recent results of Briani et al. (2012), an extension of the classical framework of Barles and Soganidis (1991) which could apply if inventories were continuous, to systems of PDEs that satisfy certain monotonicity assumption.

The rest of this paper is organized as follows. Section 2 presents the model for midprices and shows how the IT learns from innovations in midprices. Section 3 derives the IT's optimal trading strategy. Section 4 shows the behavior and financial performance of the IT's strategy and how does it compare to those of other traders who are less informed or cannot learn from market dynamics. Section 5 shows how the strategy is modified to account for adverse selection costs and Section 6 contains the main convergence results showing that the numerical scheme converges to the viscosity solution of the QVI. All proofs are collected in the appendix.

\section{Stock Price Dynamics}

Let $S_{t}^{i}$ denote the midprice of asset $i(i=1, \ldots, n, n \in \mathbb{N})$ at time $t \in[0, T]$ and assume that

$$
S_{T}^{i}=S_{0}^{i}+D^{i}
$$

where $D^{i}$ is a random variable which encodes the IT's prior belief on the future midprice distribution of asset $i$. We require that $D^{i}$ have finite second moment, and that $\mathbb{P}\left(D^{i}<\right.$ $0) \in(0,1)$ and $\mathbb{P}\left(D^{i}>0\right) \in(0,1)$, i.e., there is non-zero probability on both positive and negative outcomes to avoid arbitrage since interest rates are assumed zero. Hence, the IT may use whatever methods she has at her disposal to form a prior (such as statistical machine learning methods, price co-integration models, limit order book information, etc.) and the price dynamics we propose below will reflect the IT's belief. The structure of the probability space on which we work will be described below. 
In our set-up the IT employs market-wide information before $t=0$ to form her prior belief on the joint distribution of $D^{i}$ and, immediately after, decides how to execute a trading strategy in one or more assets between time 0 and $\bar{T}<T$. The strategy is adjusted to incorporate the arrival of new information, in the form of innovations in midprices, which the IT uses to continuously update her prior. More specifically, we assume that the midprice process is a randomized Brownian bridge $(\mathrm{rBb})$ connecting the current midprice to the future midprice, i.e.

$$
S_{t}^{i}-S_{0}^{i}=\sigma_{i} \beta_{t T}^{i}+\frac{t}{T} D^{i}
$$

where $\sigma_{i}>0$ are constants, $\beta_{t T}^{i}$ are independent ${ }^{2}$ standard Brownian bridges, independent of $D^{i}, 1 \leqslant i \leqslant n$, which satisfy

$$
\beta_{t T}^{i}=W_{t}^{i}-\frac{t}{T} W_{T}^{i},
$$

for $t \in[0, T]$, and $W_{t}^{i}$ are independent standard Brownian motions.

We work on the filtered probability space $\left(\Omega, \mathbb{P},\left\{\mathcal{F}_{t}\right\}_{0 \leqslant t \leqslant T}\right)$ with $\mathcal{F}_{t}$ generated by the processes $S_{t}^{i}$. Here the Brownian bridge $\sigma_{i} \beta_{t T}^{i}$ models fluctuations in the asset's midprice at times $t \in(t, T)$, and since the IT has access only to the filtration $\mathcal{F}_{t}$ generated by the collection of $S_{t}^{i}$, she cannot decompose $S_{t}^{i}$ into $D^{i}$ and $\beta_{t T}^{i}$ - except at $T$ where $\beta_{T T}^{i}=0$ and $S_{T}^{i}=S_{0}^{i}+D^{i}$. To simplify notation we write the vector of midprices as $\mathbf{S}_{t}=\left(S_{t}^{1}, \ldots, S_{t}^{n}\right)$, Brownian bridges as $\boldsymbol{\beta}_{t T}=\left(\beta_{t T}^{1}, \ldots, \beta_{t T}^{n}\right)$, and terminal midprice changes as $\mathbf{D}=\left(D^{1}, \ldots, D^{n}\right)$.

The algorithmic and high frequency trading literature commonly assumes the midprice follows an arithmetic Brownian motion - this is a particular case of our model if in (2) we assume that $D^{i}$ are normally distributed with mean zero and variance $\sigma_{i}^{2} T$. Further, our choice of the price process is related to Doob's $h$-transform of the underlying Brownian motions (see, e.g., Rogers and Williams (1987)). A similar model for asset prices was adopted by Brody et al. (2008) whereby a process analogous to (2) represented the information flow in the economy and asset prices are found via the conditional expectation with respect to this information, i.e. $\mathbb{E}\left[S_{0}^{i}+D^{i} \mid \mathcal{F}_{t}\right]$, rather than by (2) directly. In Brody et al. (2008), asset prices are martingales, while here the midprices are not martingales. For other applications of randomized bridges in information-based asset modeling see, e.g., Brody et al. (2009) and Macrina (2014). Moreover, similar models with applications to utility maximization were studied earlier in Baudoin (2002), Baudoin (2003), and Baudoin and Nguyen-Ngo (2004).

In the proposition below, also contained implicitly in Brody et al. (2008) and Baudoin (2002), we show the stochastic differential equation (SDE) satisfied by $S_{t}^{i}$.

\footnotetext{
${ }^{2}$ This independence assumption can easily be lifted. We impose it since, as will be shown later on, the joint distribution of the price changes induces a non-linear co-integration of asset prices while correlating the Brownian bridges will induce only an instantaneous correlation in price changes.
} 
Proposition 1. The asset's midprice process $S_{t}^{i}$ given by (2) satisfies the SDE

$$
d S_{t}^{i}=A_{i}(t, \mathbf{S}) d t+\sigma_{i} d W_{t}^{i},
$$

where $W_{t}^{i}$ are pairwise independent $\mathcal{F}_{t}$-Brownian motions, and $S_{t=0}^{i}=S_{0}^{i}$ for $1 \leqslant i \leqslant n$. Moreover,

$$
A_{i}(t, \mathbf{S})=\frac{a_{i}(t, \mathbf{S})-\left(S_{t}^{i}-S_{0}^{i}\right)}{T-t}
$$

where

$$
a_{i}(t, \mathbf{S})=\mathbb{E}\left[D^{i} \mid \mathbf{S}_{t}=\mathbf{S}\right]=\frac{\int_{\mathbb{R}^{n}} x_{i} \prod_{j=1}^{n} \exp \left(x_{j} \frac{S_{j}-S_{0}^{j}}{\sigma_{j}^{2}(T-t)}-\frac{1}{2} x_{j}^{2} \frac{t}{T \sigma_{j}^{2}(T-t)}\right) d F(\mathbf{x})}{\int_{\mathbb{R}^{n}} \prod_{j=1}^{n} \exp \left(x_{j} \frac{S_{j}-S_{0}^{j}}{\sigma_{j}^{2}(T-t)}-\frac{1}{2} x_{j}^{2} \frac{t}{T \sigma_{j}^{2}(T-t)}\right) d F(\mathbf{x})}
$$

are the $\mathcal{F}_{t}$-conditional expectations of $D^{i}$ 's, and $F=F_{\mathbf{D}}$ is the joint cumulative distribution function of the random variables $\mathbf{D}$.

This proposition shows how the trader's prior and how learning from information updates are encoded in the drift of the process (4). For instance, a trader who believes that prices are independent arithmetic Brownian motions behaves in the same way as one who uses independent normals with zero mean and variances of $\sigma_{i}^{2} T$ as his prior. Such a trader will never learn from the dynamics of the asset prices and $A(t, \mathbf{S})=0$. A second simple example is if $F$ is a Dirac mass at a single point $\boldsymbol{\delta}$. In this case, $a_{i}(t, \mathbf{S})=\delta_{i}$, and therefore, the price process is a Brownian bridge connecting the initial price $\mathbf{S}_{0}$ to the terminal price $\mathbf{S}_{0}+\boldsymbol{\delta}$. In general, the drift term $A(t, \mathbf{S})$ is non-linear and captures the (non-linear) co-integration relationship between asset prices. Throughout this paper, learning by the trader refers to the updates of the prior by observing asset prices which is not necessarily restricted to the assets the trader is taking positions in.

\subsection{Assumptions on $(\bar{T}, \mathbf{D})$ and $A(t, \mathbf{S})$}

In what follows, we consider pairs $(\bar{T}, \mathbf{D})$ so the function $A(t, S)$ is globally uniformly Lipschitz on $[0, \bar{T}] \times \mathbb{R}^{n}$. In principle $\bar{T}$ can be taken equal to $T$ as long as the global Lipschitz condition holds, for example when $D \sim N\left(0, \sigma^{2} T\right)$ so that the SDE in (4) has a unique strong solution.

Below we also consider the following modification of the model to ensure that the return of the midprice remains finite. Suppose that the prediction $D^{i}$ for midprice changes in asset $i$ has compact support. If $S_{t_{*}}^{i} \ll \inf \operatorname{supp} D^{i}$ at some time $t_{*}<\bar{T}$ but close to $\bar{T}$, the midprice would have to grow rapidly between $t_{*}$ and $\bar{T}$ in order to approach $\operatorname{supp} D^{i}$, with the rate of growth determined by (in general, the unbounded) coefficient $A_{i}(t, \mathbf{S})$, see equation (5). To address the issue of unbounded rate of growth or decline of the asset midprice, the trader may adopt 
a modification of model (2) to bound the rate of growth. More specifically, in the notation of Proposition 1, the agent assumes that the midprice $S_{t}^{i}$ satisfies

$$
d S_{t}^{i}=\tilde{A}_{i}\left(t, \mathbf{S}_{t}\right) d t+\sigma_{i} d W_{t}^{i}
$$

where

$$
\tilde{A}_{i}(t, \mathbf{S}):=\min \left\{\underline{A}_{i}, \max \left\{\bar{A}_{i}, A_{i}(t, \mathbf{S})\right\}\right\}, \quad-\infty<\underline{A}_{i}<\bar{A}_{i}<\infty, \quad i=1, \ldots, n .
$$

This modification will come into play in the proof of convergence of the numerical scheme in section 6 .

\section{Optimal trading strategy}

Innovations in midprices are used by the IT to update her prior and then to adjust her strategy by deciding how to use market and limit orders to trade in and out of positions between now and $\bar{T}<T$. Market orders guarantee immediate execution but are more expensive because the trader must pay the spread in addition to liquidity taking fees. On the other hand, limit orders do not pay fees (in some markets they even earn rebates, see Cartea and Jaimungal (2013)) but execution is not guaranteed - the trader must wait until an incoming market order fills her limit order.

Here we assume that the IT executes a strategy in a subset of $1, \ldots, k$ assets $(k \leq n)$, but nonetheless uses the intelligence she acquires from all $n$ assets to form her trading strategy. To pose the optimal control problem we require notation to describe the IT's submitted limit and market orders, cash process, accumulated inventory, and finally, the market orders sent by other participants.

The IT posts passive orders only at the touch (best bid or ask) where $\ell_{t}^{ \pm}=\left\{\ell_{t}^{1 \pm}, \ldots, \ell_{t}^{k \pm}\right\} \in$ $\{0,1\}^{k}$ denotes her decision to post a sell $(+)$ or a buy $(-)$ limit order for one unit of asset

at time $t$ with $\ell_{t}^{i \pm}=0$ meaning that there is no post. We also assume that every time there is an incoming market order, the IT's limit order is filled with probability $\mathbf{p}=\left\{p_{1}, \ldots, p_{k}\right\}$. Moreover, $\mathbf{m}_{t}^{ \pm}=\left\{m_{t}^{1 \pm}, \ldots, m_{t}^{k \pm}\right\} \in \mathbb{Z}_{+}^{k}$ counts the total number of market orders sent by the IT up until time $t$,

$$
m_{t}^{i \pm}=\sum_{k} \mathbb{1}_{\left\{\tau_{k}^{i \pm} \leqslant t\right\}}, \quad i=1, \ldots, k
$$

where + indicates a sell $\left(-\right.$ a buy), $\mathbb{1}_{\{\cdot\}}$ is the indicator function, $0<\tau_{1}^{i \pm}<\tau_{2}^{i \pm}<\ldots$ are increasing sequences of $\mathcal{H}$-stopping times representing the arrival times of the IT's executed market orders in asset $i$. The filtration $\mathcal{H}$ is the expanded filtration taking into account the arrival of other agent's market orders which fill the IT's posted limit orders and the assets' midprices. More specifically, let the counting processes $\mathbf{N}_{t}^{ \pm}=\left\{N_{t}^{1 \pm}, \ldots, N_{t}^{k \pm}\right\}$ represent the 
total number of buy and sell market orders other participants have sent in the assets which the IT trades. We assume these are Poisson processes (mutually independent and independent of the midprice processes) with intensities $\Lambda^{ \pm}>0$. Those market orders which fill the IT's posted limit orders will be denoted $\overline{\mathbf{N}}_{t}^{ \pm}=\left\{\bar{N}_{t}^{1 \pm}, \ldots, \bar{N}_{t}^{k \pm}\right\}$ and, due to the thinning property of Poisson processes, are also independent Poisson processes with intensities $\boldsymbol{\lambda}^{ \pm}=\mathbf{p} \odot \boldsymbol{\Lambda}^{ \pm}$ (where $\mathbf{x} \odot \mathbf{y}$ represents componentwise multiplication). The filtration on which strategies are adapted is given by $\mathcal{H}_{t}=\sigma\left(\left\{\mathbf{S}_{u}, \mathbf{N}_{u}^{ \pm}, \overline{\mathbf{N}}_{u}^{ \pm}\right\}_{0 \leq u \leq t}\right)$.

The number of the IT's filled limit orders in asset $i$, up to time $t$, is given by $\int_{0}^{t} \ell_{t}^{i \pm} d \bar{N}_{t}^{i \pm}$. Therefore the IT's inventory in asset $i$ at time $t$ is given by

$$
q_{t}^{i}=-\int_{0}^{t} \ell_{t}^{i+} d \bar{N}_{t}^{i+}+\int_{0}^{t} \ell_{t}^{i-} d \bar{N}_{t}^{i-}-m_{t}^{i+}+m_{t}^{i-}, \quad q_{0}^{i}=0,
$$

and we assume that the IT restricts her accumulated inventory position to be between $\underline{q}^{i} \leqslant$ $q_{t}^{i} \leqslant \bar{q}^{i}$ for all $t$. The strategy can build short positions, so $\underline{q}^{i}<0$.

The execution prices that the IT achieves for trading one unit of the asset using market orders in asset $i$ are $S_{t}^{i}-\frac{\Delta^{i}}{2}-\varepsilon^{i}$ for a sell, and $S_{t}^{i}+\frac{\Delta^{i}}{2}+\varepsilon^{i}$ for a buy, where $\varepsilon^{i}>0$ represents liquidity taking fees and $\Delta^{i}$ is the spread between the best ask and best bid which we assume to be constant.

The IT's cash process $X_{t}$ satisfies the SDE

$$
\begin{aligned}
d X_{t}=\sum_{i=1}^{k} & \left\{-\left(S_{t-}^{i}-\frac{\Delta^{i}}{2}\right) \ell_{t}^{i-} \mathbb{1}_{\left\{q_{t}^{i} \leqslant \bar{q}^{i}\right\}} d \bar{N}_{t}^{i-}+\left(S_{t-}^{i}+\frac{\Delta^{i}}{2}\right) \ell_{t}^{i+} \mathbb{1}_{\left\{q_{t}^{i} \geqslant \underline{q}^{i}\right\}} d \bar{N}_{t}^{i+}\right. \\
& \left.-\left(S_{t-}^{i}+\frac{\Delta^{i}}{2}+\varepsilon^{i}\right) \mathbb{1}_{\left\{q_{t}^{i} \leqslant \bar{q}^{i}\right\}} d m_{t}^{i-}+\left(S_{t-}^{i}-\frac{\Delta^{i}}{2}-\varepsilon^{i}\right) \mathbb{1}_{\left\{q_{t}^{i} \geqslant \underline{q}^{i}\right\}} d m_{t}^{i+}\right\}
\end{aligned}
$$

with $X_{0}=0$. The first line accounts for changes in cash due to limit orders being filled by other market participants' incoming market orders and the second line accounts for changes in cash due to the market orders that the IT submits.

Next, we turn to the IT's optimization problem who seeks the strategy $\boldsymbol{\nu}=\left\{\boldsymbol{\ell}_{t}^{ \pm}, \mathbf{m}_{t}^{ \pm}\right\}_{0 \leq t \leq \bar{T}}$ which maximizes her expected cash while penalizing running inventory. Thus, the IT's performance criteria following a given strategy $\boldsymbol{\nu}$ is given by

$$
H^{\nu}(t, X, \mathbf{S}, \mathbf{q})=\mathbb{E}_{t, X, \mathbf{S}, \mathbf{q}}\left[X_{\bar{T}}+\sum_{i=1}^{k}\left\{\psi^{i}\left(q_{\bar{T}}^{i}, S_{\bar{T}}^{i}\right)-\phi^{i} \int_{0}^{\bar{T}}\left(q_{u}^{i}\right)^{2} d u\right\}\right]
$$

where $\mathbb{E}_{t, X, \mathbf{S}, \mathbf{q}}[\cdot]$ denotes expectation conditional on $X_{t-}=X, \mathbf{S}_{t-}=\mathbf{S}$, and $\mathbf{q}_{t-}=\mathbf{q}$ and $\psi$ 
represents the terminal liquidation costs / value

$$
\psi^{i}(q, S)=q\left(S-\left(\frac{\Delta^{i}}{2}+\varepsilon^{i}\right) \operatorname{sgn}(q)-\alpha^{i} q\right) .
$$

The IT's value function is defined as

$$
H(t, X, \mathbf{S}, \mathbf{q})=\sup _{\boldsymbol{\nu} \in \mathcal{A}} H^{\boldsymbol{\nu}}(t, X, \mathbf{S}, \mathbf{q})
$$

As is usual, we seek Markov optimal controls (and under quite general assumptions, the optimum is indeed Markov ).

In the right-hand side of the performance criteria (12) there are three components. The first is terminal cash. The second component captures the costs that the IT incurs at the terminal date $\bar{T}$ when liquidating $q_{\bar{T}}$. The terminal inventory is unwound with a market order so picks up the costs of crossing the spread, as well as any other costs including liquidity taking fees and (linear) market impact, both of which are captured by the parameter $\alpha^{i}>0$. And the third component is a running penalty that the IT imposes to control her exposure to inventory risk, see Cartea and Jaimungal (2015b) and Cartea et al. (2013) where the authors demonstrate that this type of running penalty can be viewed as arising from model uncertainty (ambiguity aversion) in the drift of the asset's midprice process.

Proposition 2. The value function $H$ admits the representation

$$
H(t, X, \mathbf{S}, \mathbf{q})=X+\sum_{i=1}^{k} q^{i} S^{i}+g(t, \mathbf{S}, \mathbf{q})
$$

where $g$ is the unique solution to the QVI

$$
\begin{aligned}
& 0=\max \left\{\partial_{t} g\right.+\sum_{i=1}^{k}\left\{\frac{1}{2} \sigma_{i}^{2} \partial_{S^{i} S^{i}} g+A_{i}(t, \mathbf{S})\left(q^{i}+\partial_{S^{i}} g\right)-\phi^{i}\left(q^{i}\right)^{2}\right\} \\
&+\sum_{i=k+1}^{n}\left\{\frac{1}{2} \sigma_{i}^{2} \partial_{S^{i} S^{i}} g+A_{i}(t, \mathbf{S}) \partial_{S^{i}} g\right\} \\
&+\sum_{i=1}^{k} \mathbb{1}_{q^{i}<\bar{q}^{i}} \lambda^{i-} \max _{\ell \in\{0,1\}}\left[\frac{\Delta^{i}}{2} \ell+g\left(t, \mathbf{S}, \mathbf{q}+\boldsymbol{\delta}^{i} \ell\right)-g(t, \mathbf{S}, \mathbf{q})\right] \\
&+\sum_{i=1}^{k} \mathbb{1}_{q^{i}>\underline{q}^{i}} \lambda^{i+} \max _{\ell \in\{0,1\}}\left[\frac{\Delta^{i}}{2} \ell+g\left(t, \mathbf{S}, \mathbf{q}-\boldsymbol{\delta}^{i} \ell\right)-g(t, \mathbf{S}, \mathbf{q})\right] ; \\
&\left.\max _{\boldsymbol{\xi} \in \mathcal{D}(\mathbf{q})}\left\{-\sum_{i=1}^{k}\left(\frac{\Delta^{i}}{2}+\varepsilon^{i}\right)\left|\xi^{i}\right|+g(t, \mathbf{S}, \mathbf{q}+\boldsymbol{\xi})-g(t, \mathbf{S}, \mathbf{q})\right\}\right\},
\end{aligned}
$$

$\boldsymbol{\delta}^{i}$ is a $k$-vector with $\delta^{i j}=0$ for $j \neq i$ and $\delta^{i i}=1$, the set

$$
\mathcal{D}(\mathbf{q})=\otimes_{i=1}^{k}\left\{-\mathbb{1}_{q^{i}>\underline{q}^{i}}, 0, \mathbb{1}_{q^{i}<\bar{q}^{i}}\right\}
$$


and the QVI is subject to the terminal condition

$$
g(\bar{T}, \mathbf{S}, \mathbf{q})=-\sum_{i=1}^{k}\left(\left(\frac{\Delta^{i}}{2}+\varepsilon^{i}\right)\left|q^{i}\right|+\alpha^{i}\left(q^{i}\right)^{2}\right), \quad \underline{q}^{i} \leqslant q^{i} \leqslant \bar{q}^{i} .
$$

In (16) the first line represents the flow of asset midprices and the updates of the priors in the assets in which the IT trades, while the second line represents the flow of asset midprices and the updates of the priors in the other assets. The third and fourth lines represent the change in the value function due to execution of the agent's posted limit orders. The last line corresponds to the execution of market orders, and $\mathcal{D}(\mathbf{q})$ is the set of allowed market order executions which respect the inventory limits imposed by the IT.

To solve the QVI numerically, and obtain a unique $g$, we impose a grid in $\mathbf{S}$ with boundaries $\underline{S}^{i} \leq S^{i}<\bar{S}^{i}$ and the additional boundary conditions

$$
\partial_{S^{i} S^{i}} g\left(t, \ldots, \underline{S}^{i}, \ldots, \mathbf{q}\right)=0, \quad \partial_{S^{i} S^{i}} g\left(t, \ldots, \bar{S}^{i}, \ldots, \mathbf{q}\right)=0
$$

for all $0<t<\bar{T}, \underline{q}^{i} \leqslant q^{i} \leqslant \bar{q}^{i}$.

These constraints reflect the intuition that the trader's actions have a negligible effect on her profit when the midprice $S$ is far from the support of the prediction $D$. Hence, for a large $|S|$, the value function $H$ is approximately linear and in view of the ansatz (15), we impose these boundary conditions on $g$. Furthermore, we observe that the optimal trading strategy does not change as $|\bar{S}|,|\underline{S}| \rightarrow \infty$. In particular, we set $\left|\bar{S}^{i}\right|=S_{0}^{i}+\gamma \sigma \sqrt{T}$ and $\left|\bar{S}^{i}\right|=S_{0}^{i}-\gamma \sigma \sqrt{T}$ and take $\gamma=5$ as larger values result in the same optimal strategies.

Learning from midprice dynamics is impaired by the volatility of prices. When $\sigma$ increases the IT becomes less confident about her predictions. Indeed, if volatility increases past a certain critical level, processing information does not give her any advantage and her strategy becomes the same as that of a trader who sees the asset evolve like an arithmetic Brownian motion.

\section{Behavior and performance of the optimal trading strategy}

In this section we use simulations to analyze the behavior and financial performance of the IT's strategy. The details of the numerical scheme and its convergence are provided in section 6. Here we focus on the numerical results and their financial interpretation. To show how much being better informed is worth, we compare her strategy to that of two other type of traders:

i) An uniformed trader who does not have the capability to form a prior or learn from price innovations and therefore assumes that the midprice dynamics are given by an arithmetic Brownian motion - we label him the uniformed trader (UT). In this case, the UT's strategy 
becomes that of an investor who is providing liquidity similar to the market maker models described in the extant literature, see for example Avellaneda and Stoikov (2008), and Chapter 10 in Cartea et al. (2015).

ii) The other is a trader who has an uniformed prior, but is able to learn from market dynamics - we label him the uninformed learner (UL). His prior is symmetric around the current midprice, but it is not necessarily independent normal with mean zero and variance $\sigma_{i}^{2} T$ as is the UT's. As a result, the UL is able to learn from the dynamics of the midprice.

To gain insights into the performance of the traders' strategies, we first show results when they trade and learn from only one asset, and then show results for $n=2$ where traders learn from two assets and take positions in only one of them.

\subsection{Learning from, and trading in, one asset}

All three traders assume that the asset dynamics are given by (4). What distinguishes them is their prior distribution on the value of the asset at time $T$. In particular, IT and UL assume that $D^{1}$ is a mixture of Gaussians, and has probability density function (pdf)

$$
\mathbb{P}\left(D^{1} \in[x, x+d x)\right)=\left\{p_{u} \phi\left(x, \delta_{u}, \mu_{u}\right)+p_{d} \phi\left(x, \delta_{d}, \mu_{d}\right)\right\} d x, \quad x \in \mathbb{R},
$$

where $\phi(x, \delta, \mu)$ is a Normal pdf with mean $\delta$ and standard deviation $\mu, 0 \leqslant S_{0}^{1}+\delta_{d} \leqslant S_{0}^{1}+\delta_{u}$ and $p_{u}+p_{d}=1$. More specifically, for the numerical experiments below we choose

- IT's prior is that the pdf of $D^{1}$ is as in (19) with $\delta_{u}=0.02$ and $\delta_{d}=-0.02$, with probabilities $p_{u}=0.8$ and $p_{d}=0.2, \mu_{u}=\mu_{f}=7 \cdot 10^{-2}$,

- UL's prior is that the pdf of $D^{1}$ is as in (19) with $\delta_{u}=0.02$ and $\delta_{d}=-0.02$, with probabilities $p_{u}=0.5$ and $p_{d}=0.5, \mu_{u}=\mu_{d}=7 \cdot 10^{-2}$, and finally

- UT believes that $D^{1} \sim N\left(0, \sigma_{1} \sqrt{T}\right)$. This choice results in the UT assuming the midprice is a Brownian motion with volatility $\sigma_{1}$ so that $S_{t}^{1}=S_{0}^{1}+\sigma_{1} W_{t}^{1}$.

In the simulations, we assume that the midprice follows the $\mathrm{rBb}(2)$ where at time $T$ the asset's midprice will increase to $S_{0}^{1}+\delta_{u}$, where $S_{0}^{1}=1$ and $\delta_{u}=0.02$ (with probability 1 ), and $\sigma_{1}=0.01$. We assume that all three traders know the correct $\sigma_{1}$ parameter and we see that in this set-up although the IT and UL 'learn' from market dynamics using the same mechanism, the IT has a more accurate prior than that of the UL. Market orders from other participants which fill the traders posted limit orders arrive with intensities $\lambda^{ \pm}=30$. In the simulations the actions of the traders are small enough not to alter the dynamics of the limit order book or the midprice. Finally, the other model parameters are: $\bar{T}=1, T=1.1, \Delta^{1}=10^{-2}, \varepsilon^{1}=\Delta^{1} / 20$, $\alpha^{1}=5 \cdot 10^{-3}, \underline{q}^{1}=-20, \bar{q}^{1}=20$, and $\phi^{1}=0$. 


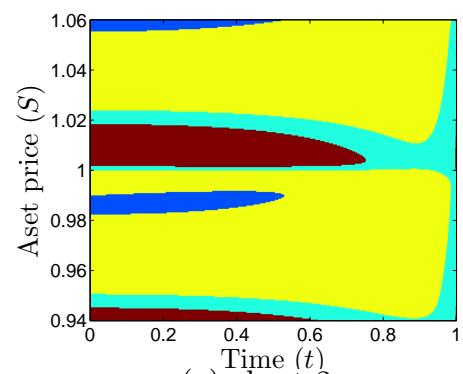

(a) short 2

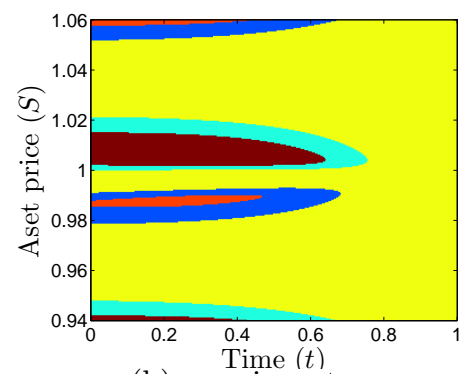

(b) zero inventory

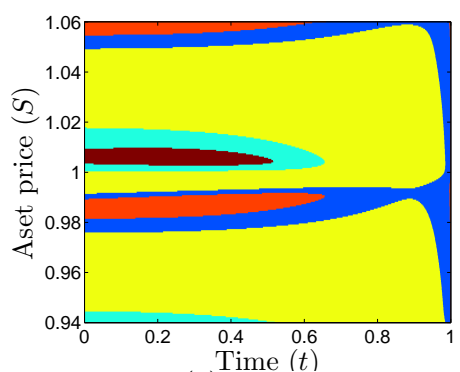

(c) long 2

Figure 1: The IT trading strategy. Maroon: post a buy market order. Orange: post a sell market order. Cyan: post a buy limit order. Blue: post a sell limit order. Yellow: post both buy and sell limit orders. Dark Blue: do not post any orders. Note: near maturity $t=1$, there is a dark blue sliver when $q=0$, a maroon sliver when $q=-2$ and an orange sliver when $q=2$.

Figure 1 shows the optimal strategy the IT uses to trade in the asset at all points in time for a range of prices and for three inventory levels. As the agent moves from a short position to a long position, the buy (sell) regions - both for sending market orders and for positing limit orders - shrink (expand). There is a clear asymmetry in the strategy due to the IT having a weight of 0.8 placed on the asset price increasing to $\$ 1.02$. From the starting asset price of $\$ 1.00$, however, the agent does not immediately execute buy market orders, rather there is a thin region around which the agent posts either buy limit orders only or both buy and sell limit orders. Only when the price moves slightly upwards does she execute a buy market order. On the other hand, if the price moves down sufficiently she cancels the buy limit order and posts only sell limit orders. If the price moves even further down quickly, then (when $q=0$ or 2) she executes a sell market order. If prices move upwards sufficiently quickly, the agent also executes sell market orders since she has the view that the price cannot end higher than $\$ 1.02$.

For comparison purposes, we also show in Figure 2 the optimal strategy when the agent only posts limit orders. When $q=0$, the regions where the agent posts only buy or sell limit orders shrink, and the region where she posts both type of orders is expanded. The reason is that she is not able to fully unwind using market orders, and is therefore more conservative on taking directional bets. When $q=+2(q=-2)$ the sell (buy) limit order region expands to increase the likelihood that she unwinds her position by the end of the trading horizon.

Figure 3 shows the strategies of the three traders for the same midprice path. On the lefthand $y$-axis we show the midprice and on the right-hand $y$-axis we show accumulated inventory, the $x$-axis records the time from the start of the strategy up until time $\bar{T}=1$. In the figure, stars denote the arrival of other participant's market orders, the solid circles denote the trader's buy market orders, and the empty circles denote the trader's sell market orders. The green (red) line shows the times when the trader is posting buy (sell) limit orders. Furthermore, we 


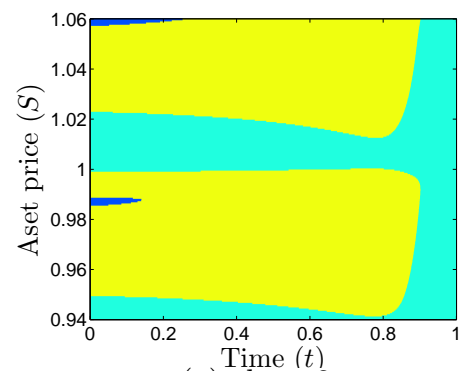

(a) short 2

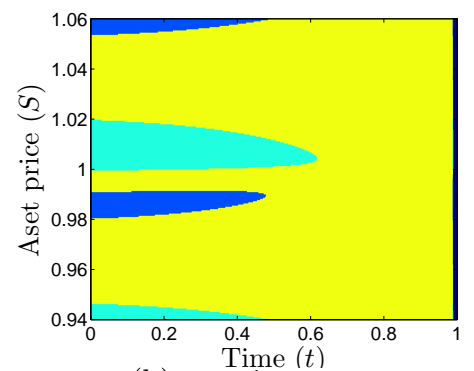

(b) zero inventory

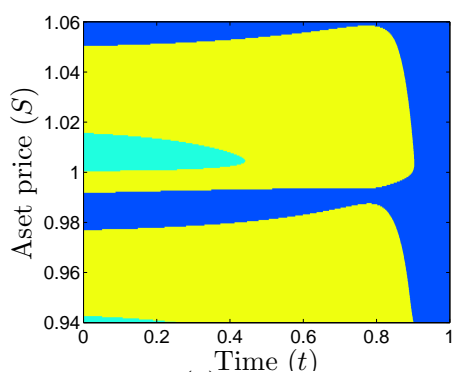

(c) long 2

Figure 2: The IT is posting limit orders only (cannot execute market orders). Cyan: post a buy limit order. Blue: post a sell limit order. Yellow: post both buy and sell limit orders. Dark Blue: do not post any limit orders. Note: near maturity $t=1$, there is a dark blue sliver when $q=0$.

see that the midprice is the path that starts at $S_{0}^{1}=1$ and ends at $S_{1.1}^{1}=1.02$ so that at $t=1$ the midprice is very close to 1.02. Around the midprice path we see the trader's limit orders who posts at the best bid and best ask, which are $\pm \frac{1}{2} \Delta^{1}$ away from the midprice.

In panel (a) we see the strategy followed by the IT. Clearly, at the beginning of the strategy the IT submits a number of market buy orders, as well as predominantly submitting one-sided buy limit orders, to build up inventories because her prior is that the midprice will increase. As time evolves and the IT has built a long inventory, the strategy stops relying on market orders and concentrates on two-sided limit orders to control inventories whilst earning the spread. Nearer the end of the strategy, the IT stops posting limit orders on the buy side of the book and unwinds inventories using both limit and market sell orders.

In contrast, we see the strategy adopted by the UT. Panel (b) shows how the UT's inability to learn stops him from behaving as aggressively as the IT. At no point does the UT use market orders to build or unwind inventories. In fact, the UT devises a strategy where inventories mean revert to zero very quickly to avoid exposure to inventory risk. Finally, the strategy followed by the UL is somewhere in between that of the IT and UT, see panel (c).

The strategy of both the IT and UL is distinct from that of a market maker, who is expected to continuously post two-sided quotes (not necessarily at the touch). Even though between the start and final date of the strategy the IT and UL post on both sides of the limit order book, this is a result of their information updates which induce the strategy to control inventory exposure. The UT's strategy is closer to that of a market maker because he can only make money from the spread since he has no directional view on the market.

The mix between limit and market orders used by the traders depends on how confident they are about their prior as well as their ability to extract information from midprice dynamics. Even though in the simulations we assume that the midprice ends at time $T=1.1$, and the strategy closes at $\bar{T}=1$, the Brownian bridge adds noise to the path and the IT and UL need 


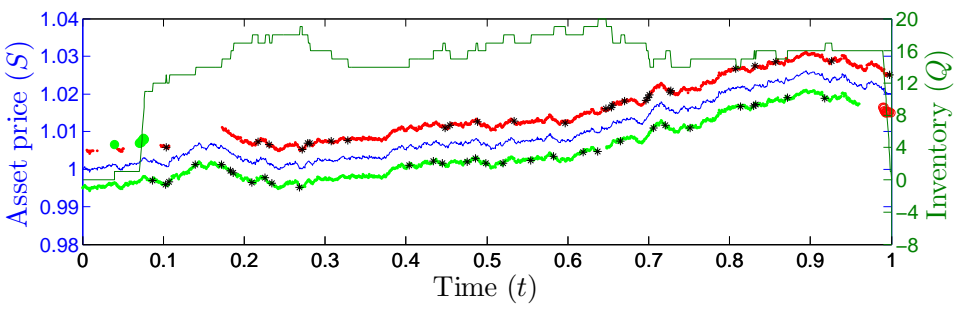

(a) IT

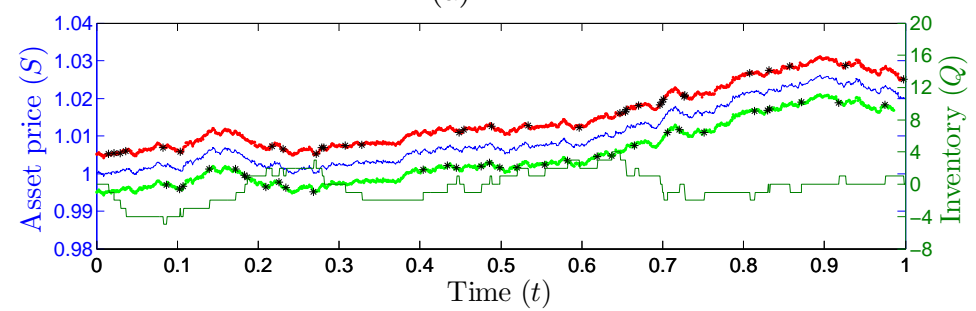

(b) UT

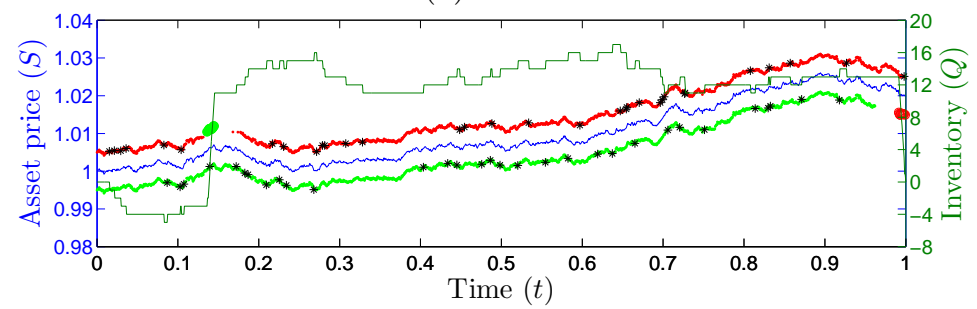

(c) UL

Figure 3: Sample path of midprice (blue), inventories (thin green), and trading strategy (thick green and red lines for when limit orders are posted, and green and red circles when market orders are executed).

to update their prior and decide whether to send market orders and/or to post one-sided or two-sided limit orders and wait until they cancel them or are filled. In Figure 4 we show the average number, for $10^{4}$ midprice paths, of market and filled limit orders for the three traders. To calculate the average number of orders we divide the time interval $[0,1]$ into 20 subintervals, and compute within each subinterval the average number of filled limit orders and the average number of submitted market orders. If a limit order is posted but not filled, then it is not taken into account in these averages.

The figure shows that the strategy used by the traders is not only based on a directional view of the market trend (as shown by the market orders they employ), but it also executes round-trip trades during the life of the strategy. It is clear that the lesser the quality of the information used by the trader, the closer the strategy to that of a market maker who aims at making the spread and not to bet on any particular direction the asset price might take, see panel (b). 


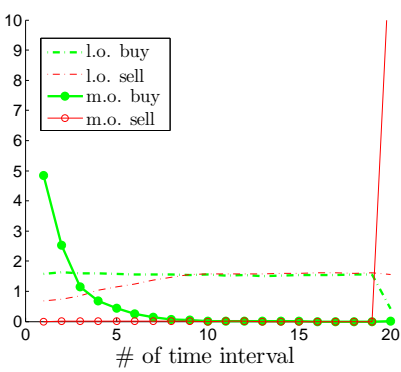

(a) IT trader

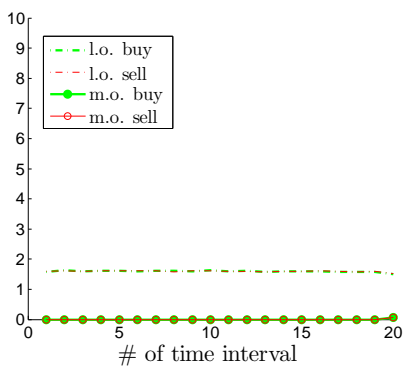

(b) UT trader

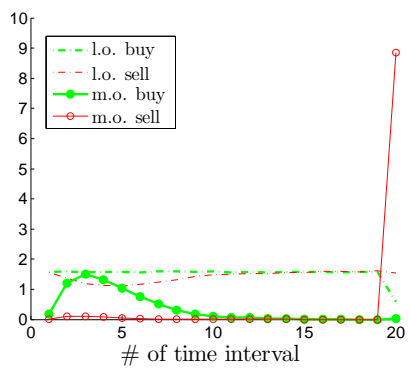

(c) UL trader

Figure 4: Average number of posted market orders (circles) and filled limit orders (dash).

What is the incremental value from being better informed? In Figure 5 we show the risk-reward profiles for the three traders for different values of the maximum and minimum inventory that they are willing to bear (panel (a)), and for different values of the running inventory penalty $\phi$ (panel (b)). Clearly, the IT outperforms the other two traders in both expected and standard deviation of her strategy's profit and loss (P\&L). The financial gains from this superiority are intensified if the traders are allowed to build larger inventory positions, but we recall that there is a limit to these gains since we are assuming that the traders' strategies are small enough not to move the market. ${ }^{3}$ Furthermore, as panel (b) shows, if the running inventory penalty is increased, the gains from better information decrease because the higher the penalty, the more conservative is the strategy as a result of exerting tighter controls on the inventory position, see Cartea and Jaimungal (2015b).

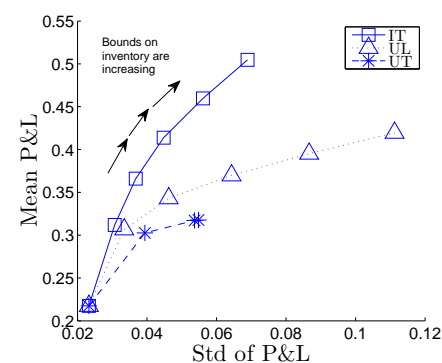

(a) $\bar{q}^{1}=-\underline{q}^{1}=1,5,10, \ldots, 20,25$.

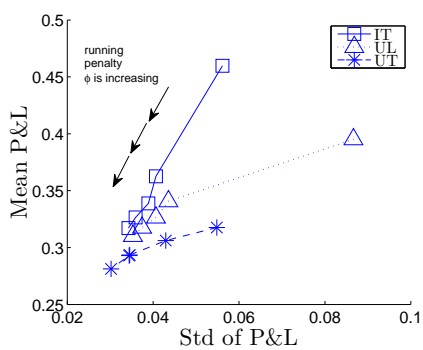

(b) $\phi^{1}=0,10^{-3}, \ldots, 4 \cdot 10^{-3}$.

Figure 5: Risk-return profiles for different constraints on inventory and levels of the running inventory penalty.

\footnotetext{
${ }^{3}$ One issue we do not discuss here is the costs associated to acquiring information. Indeed, traders must take into account the costs of processing and acquiring information and compare these to the profits that this information generate.
} 


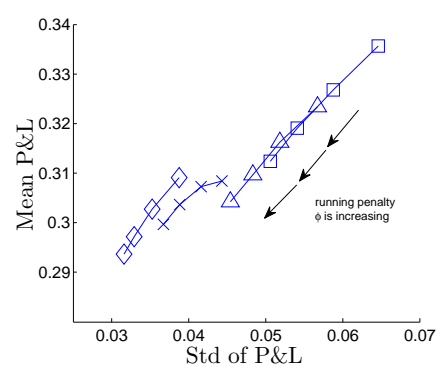

Figure 6: Squares $\square$ : trading in high volatility asset and learning from both high and low volatility assets. Triangles $\triangle$ : trading in high volatility asset without learning. Diamonds $\diamond$ : trading in low volatility asset without learning. Exes $\times$ : trading in low volatility asset and learning from both high and low volatility assets.

\subsection{Learning from two assets and trading in one asset}

Here we illustrate how information from other assets is employed to trade in another asset. Assume that there are two assets with midprices $S_{t}^{1}$ and $S_{t}^{2}$ which satisfy (4) with $\sigma_{1}=0.02$ and $\sigma_{2}=0.01$. In addition, there are common factors driving their midprice movements which are captured by the joint distribution of $D^{1}$ and $D^{2}$ assumed to be a two-dimensional Gaussian mixture model with pdf

$$
\begin{aligned}
& \mathbb{P}\left(\left(D^{1}, D^{2}\right) \in\left[x_{1}, x+d x_{1}\right) \times\left[x_{2}, x+d x_{2}\right)\right) \\
& \quad=p_{d d} \phi\left(x^{1}, x^{2} ; \delta_{d}^{1}, \delta_{d}^{2}, \mu_{d}^{1}, \mu_{d}^{2}\right)+p_{d u} \phi\left(x^{1}, x^{2} ; \delta_{d}^{1}, \delta_{u}^{2}, \mu_{d}^{1}, \mu_{u}^{2}\right) \\
& \quad+p_{u d} \phi\left(x^{1}, x^{2} ; \delta_{u}^{1}, \delta_{d}^{2}, \mu_{u}^{1}, \mu_{d}^{2}\right)+p_{u u} \phi\left(x^{1}, x^{2} ; \delta_{u}^{1}, \delta_{u}^{2}, \mu_{u}^{1}, \mu_{u}^{2}\right), \quad\left(x^{1}, x^{2}\right) \in \mathbb{R}^{2},
\end{aligned}
$$

where $\phi\left(x^{1}, x^{2} ; \delta^{1}, \delta^{2}, \mu^{1}, \mu^{2}\right)$ denotes the (independent) bi-variate normal pdf with mean $\left(\delta^{1}, \delta^{2}\right)$ and standard deviation $\left(\mu^{1}, \mu^{2}\right)$, and $p_{d d}, p_{d u}, p_{u d}, p_{u u}$ and $\delta_{d}^{1}, \delta_{u}^{1}, \delta_{d}^{2}, \delta_{u}^{2}$ are provided in Table 1. We further assume $\bar{T}=1, T=1.1, \Delta^{1,2}=10^{-2}, \varepsilon^{1,2}=\Delta^{1,2} / 20, \alpha^{1,2}=5 \cdot 10^{-3}, \underline{q}^{1,2}=-20$, $\bar{q}^{1,2}=20, \mu^{1}=\mu^{2}=7 \cdot 10^{-2}$ and $\phi^{1,2}=0$.

\begin{tabular}{cccc}
\hline \hline & & \multicolumn{2}{c}{$D^{1}$} \\
\cline { 3 - 4 } & & $\delta_{d}^{1}=-0.03$ & $\delta_{u}^{1}=+0.03$ \\
\hline \multirow{2}{*}{$D^{2}$} & $\delta_{d}^{2}=-0.02$ & 0.45 & 0.05 \\
& $\delta_{u}^{2}=+0.02$ & 0.05 & 0.45 \\
\hline \hline
\end{tabular}

Table 1: Joint probability distribution of $D^{1}$ and $D^{2}$.

If the IT focuses only on one asset, then the probabilities assigned to the asset ending at $S_{0}^{i}+D^{i}$ are $p_{u}=p_{d}=0.5$ for $i=1,2$. However, if the IT is able to learn from the dynamics of both assets then she will be able to make better predictions of the midprice at a future date. To show the incremental gains due to learning from both assets and trading in one we compare the P\&L resulting from strategies where the IT learns from one or two assets and trades in one. Figure 6 shows the risk-reward profile for the four strategies for different values of the running inventory penalty. Clearly, learning from both assets increases the profitability of the 


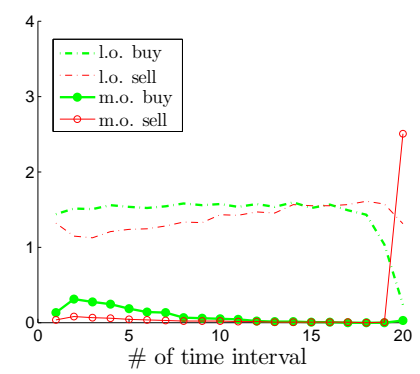

(a) $\bar{q}^{1}=-\underline{q}^{1}=1,5,10, \ldots, 20,25$.

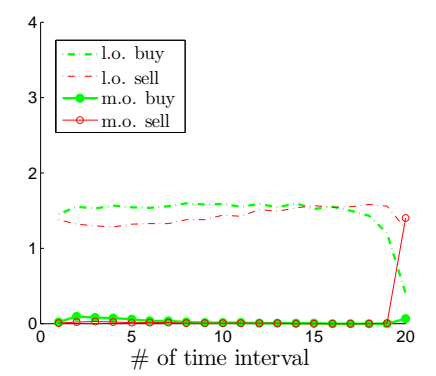

(b) $\phi^{1}=0,10^{-3}, \ldots, 4 \cdot 10^{-3}$.

Figure 7: Average number of posted market orders (circles) and filled limit orders (dash) when trader learns from both assets.

strategy.

Moreover, Figure 7 shows the average number of market and filled limit orders for $10^{4}$ midprice paths when the trader takes a position in one asset but learns from both. Panel (a) (panel (b)) shows the average orders when trading in the high (low) volatility asset. The figure shows that the strategy for both assets relies heavily on making the spread using two-sided limit orders with a slight tilt to more buy limit orders at the beginning because the prediction is that the asset price will increase.

\section{Incorporating Adverse Selection}

Thus far we have not incorporated the impact that other trader's actions have on the midprice process. It is well known that when market orders arrive they tend to push prices in the direction of trades and this feedback effect should be taken into account (see, e.g., Lipton et al. (2013), Bechler and Ludkovski (2014), Cartea and Jaimungal (2014), Cartea et al. (2015)). Such actions can result in the IT being at least locally adversely selected - e.g., after a limit order purchase, the price goes down because the IT's limit order was matched by a very fast informed trader who makes profits from ultra-fast short-term predictions. To account for such effects we consider the following variant of the model and assume that prices satisfy the SDE

$$
d S_{t}^{i}=A(t, \mathbf{S}) d t+\sigma^{i} d W_{t}^{i}+\eta^{i}\left(d N_{t}^{i+}-d N_{t}^{i-}\right)
$$

where $\eta^{i}>0$ are price impact constants (these can be made random with little difficulty), and recall that $\mathbf{N}_{t}^{ \pm}$are Poisson processes counting the number of buy/sell market orders posted by the other market participants up to time $t$. Here, for simplicity, we assume that the IT's posted limit orders are filled with probability one $\left(p^{i}=1\right)$ if a market order arrives while they are posted. It is not too difficult to lift this assumption, but in the interest of keeping the discussion concise we assume that the limit orders are always at the front of the queue. Such 


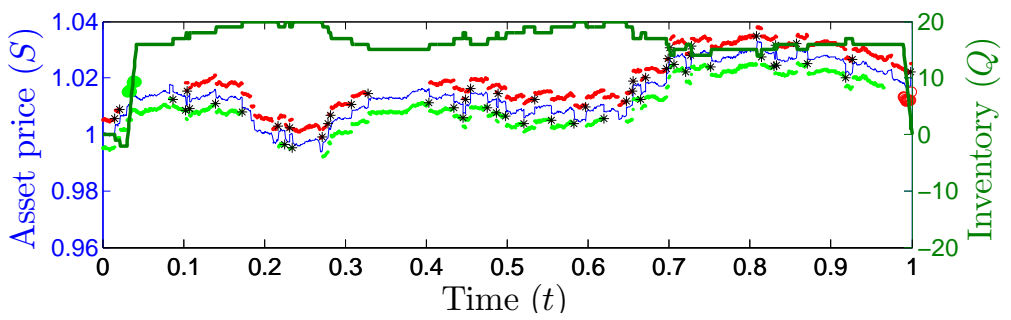

Figure 8: Sample path of midprice (blue), inventories (dark green), and trading strategy (light green and red lines for when limit orders are posted, and green and red circles when market orders are executed) in the presence of adverse selection.

a model is similar in spirit to the one adopted in Cartea and Jaimungal (2015b) where the authors show that market makers who can post anywhere in the limit order book modify their strategies by posting deeper into the book to account for adverse selection costs.

The IT still seeks the value function (14) and the ansatz $H(t, X, \mathbf{S}, \mathbf{q})=X+\sum_{i=1}^{k} q^{i} S^{i}+$ $g(t, \mathbf{S}, \mathbf{q})$ still hods; however, the QVI satisfied by $g$ is modified to

$$
\begin{aligned}
& 0=\max \left\{\partial_{t} g\right.+\sum_{i=1}^{k}\left\{\frac{1}{2} \sigma_{i}^{2} \partial_{S^{i} S^{i}} g+A_{i}(t, \mathbf{S})\left(q^{i}+\partial_{S^{i}} g\right)-\phi^{i}\left(q^{i}\right)^{2}\right\} \\
&+\sum_{i=k+1}^{n}\left\{\frac{1}{2} \sigma_{i}^{2} \partial_{S^{i} S^{i}} g+A_{i}(t, \mathbf{S}) \partial_{S^{i}} g\right\} \\
&+\sum_{i=1}^{k} \mathbb{1}_{q^{i}<\bar{q}^{i}} \lambda^{i-} \max _{\ell \in\{0,1\}}\left[\left(\frac{\Delta^{i}}{2}-\eta^{i}\right) \ell+g\left(t, \mathbf{S}, \mathbf{q}+\boldsymbol{\delta}^{i} \ell\right)-g(t, \mathbf{S}, \mathbf{q})\right] \\
&+\sum_{i=1}^{k} \mathbb{1}_{q^{i}>\underline{q}^{i}} \lambda^{i+} \max _{\ell \in\{0,1\}}\left[\left(\frac{\Delta^{i}}{2}-\eta^{i}\right) \ell+g\left(t, \mathbf{S}, \mathbf{q}-\boldsymbol{\delta}^{i} \ell\right)-g(t, \mathbf{S}, \mathbf{q})\right] ; \\
&\left.\max _{\boldsymbol{\xi} \in \mathcal{D}(\mathbf{q})}\left\{-\sum_{i=1}^{k}\left(\frac{\Delta^{i}}{2}+\varepsilon^{i}\right)\left|\xi^{i}\right|+g(t, \mathbf{S}, \mathbf{q}+\boldsymbol{\xi})-g(t, \mathbf{S}, \mathbf{q})\right\}\right\} .
\end{aligned}
$$

The key change is that in lines three and four the gains the IT makes from her posted limit order being filled (relative to the midprice) decreases by $\eta^{i}$ to $\frac{\Delta^{i}}{2}-\eta^{i}$.

Figure 8 shows actions of the IT when other participants' market orders affect midprices as in (21). The price impact parameter is $\eta^{1}=3 \cdot 10^{-3}$. Notice that in this case the IT initially posts limit orders symmetrically (in contrast to when there was no market impact, compare with Figure 3(a)) because the presence of adverse selection makes the IT wait until the price is pushed sufficiently upwards (in this case by the arrival of two market buy orders which have positive price impact) before modifying her strategy to become directional.

\section{Convergence Results}

We now address the problem of finding numerically the optimal trading strategy when the asset midprices satisfy (7). The trader's inventory $q_{t}$, cash $X_{t}$, and value function $\tilde{H}(t, X, \mathbf{S}, \mathbf{Q})$ 
are defined as in Section 3 but with $\mathbf{S}_{t}=\left(S_{t}^{1}, \ldots, S_{t}^{n}\right)$ being the solution of (7). Then $\tilde{H}$ admits the representation

$$
\tilde{H}(t, X, \mathbf{S}, \mathbf{q})=X+\sum_{i=1}^{k} q^{i} S^{i}+\tilde{g}(t, \mathbf{S}, \mathbf{q})
$$

where $\tilde{g}$ satisfies the QVI

$$
\begin{aligned}
& 0=\max \left\{\partial_{t} \tilde{g}\right.+\sum_{i=1}^{k}\left\{\frac{1}{2} \sigma_{i}^{2} \partial_{S^{i} S^{i}} \tilde{g}+\tilde{A}_{i}(t, \mathbf{S})\left(q^{i}+\partial_{S^{i}} \tilde{g}\right)-\phi^{i}\left(q^{i}\right)^{2}\right\} \\
&+\sum_{i=k+1}^{n}\left\{\frac{1}{2} \sigma_{i}^{2} \partial_{S^{i} S^{i}} \tilde{g}+\tilde{A}_{i}(t, \mathbf{S}) \partial_{S^{i}} \tilde{g}\right\} \\
&+\sum_{i=1}^{k} \mathbb{1}_{q^{i}<\bar{q}^{i}} \lambda^{i-} \max _{\ell \in\{0,1\}}\left[\frac{\Delta^{i}}{2} \ell+\tilde{g}\left(t, \mathbf{S}, \mathbf{q}+\boldsymbol{\delta}^{i} \ell\right)-(1+\kappa) \tilde{g}(t, \mathbf{S}, \mathbf{q})\right] \\
&+\sum_{i=1}^{k} \mathbb{1}_{q^{i}>\underline{q}^{i}} \lambda^{i+} \max _{\ell \in\{0,1\}}\left[\frac{\Delta^{i}}{2} \ell+\tilde{g}\left(t, \mathbf{S}, \mathbf{q}-\boldsymbol{\delta}^{i} \ell\right)-(1+\kappa) \tilde{g}(t, \mathbf{S}, \mathbf{q})\right] \\
&\left.\max _{\boldsymbol{\xi} \in \mathcal{D}(\mathbf{q})}\left\{-\sum_{i=1}^{k}\left(\frac{\Delta^{i}}{2}+\varepsilon^{i}\right)\left|\xi^{i}\right|+\tilde{g}(t, \mathbf{S}, \mathbf{q}+\boldsymbol{\xi})-(1+\kappa) \tilde{g}(t, \mathbf{S}, \mathbf{q})\right\}\right\} \\
& \tilde{g}(\bar{T}, \mathbf{S}, \mathbf{q})=-\sum_{i=1}^{k}\left(\left(\frac{\Delta^{i}}{2}+\varepsilon^{i}\right)\left|q^{i}\right|+\alpha^{i}\left(q^{i}\right)^{2}\right), \quad \underline{q}^{i} \leqslant q^{i} \leqslant \bar{q}^{i},
\end{aligned}
$$

where $\kappa \downarrow 0$ is a robustness parameter.

We seek bounded viscosity solutions $\tilde{g}$ of (24) satisfying the terminal conditions (25). If the trader restricts herself to posting limit orders only, then there exists a classical bounded solution $\tilde{g} \in C\left([0, \bar{T}] \times \mathbb{R}^{n}\right) \cap C^{1,2}\left((0, \bar{T}) \times \mathbb{R}^{n}\right)$ of $(24)$, see e.g. Becherer and Schweizer (2005).

Below we introduce a finite difference scheme that produces discrete approximations to the viscosity solution $g$ of $(16)$ on $[0, \bar{T}] \times \mathbb{R}^{n}$. In order to prove that it converges, we use the result of Briani et al. (2012), an extension of the classical framework of Barles and Soganidis (1991) to systems of PDEs satisfying certain monotonicity assumptions.

\subsection{Preliminaries}

To simplify notation, in what follows we restrict ourselves to the case of a single asset, i.e. $n=1$. The general case of $n \geqslant 2$ assets is treated similarly.

Consider the system of equations

$$
F_{q}\left(\tau, S, u, \partial_{\tau} u, \partial_{S} u, \partial_{S S} u\right)=0
$$

( $u$ is the solution of the QVI which we seek) with initial conditions

$$
u_{q}(0, S)=-\left(\frac{\Delta}{2}+\varepsilon\right)|q|+\alpha(q)^{2}, \quad \underline{q} \leqslant q \leqslant \bar{q},
$$


where the vectors $u=\left(u_{q}\right), \partial_{\tau} u=\left(\partial_{\tau} u_{q}\right), \partial_{S} u=\left(\partial_{S} u_{q}\right), \partial_{S S} u=\left(\partial_{S S} u_{q}\right)$, and the function

$$
\begin{aligned}
& F_{q}(\tau, S, u, c, p, X)=\max \left\{c_{q}\right.-\frac{1}{2} \sigma^{2} X_{q}+\tilde{A}(\bar{T}-\tau, S)\left(q-p_{q}\right)-\phi q^{2} \\
&+ \mathbb{1}_{q<\bar{q}} \lambda^{-} \max _{\ell \in\{0,1\}}\left[\frac{\Delta}{2} \ell+(1+\kappa) u_{q}-u_{q+\ell}\right] \\
&+\mathbb{1}_{q>\underline{q}} \lambda^{+} \max _{\ell \in\{0,1\}}\left[\frac{\Delta}{2} \ell+(1+\kappa) u_{q}-u_{q-\ell}\right] ; \\
&\left.\max _{\xi \in\left\{-\mathbb{1}_{q>\underline{q}}, 0, \mathbb{1}_{q<\bar{q}}\right\}}\left\{-\left(\frac{\Delta}{2}+\varepsilon\right)|\xi|+(1+\kappa) u_{q}-u_{q+\xi}\right\}\right\},
\end{aligned}
$$

for vectors $c=\left(c_{q}\right), p=\left(p_{q}\right)$, and $X=\left(X_{q}\right)$.

The next result ensures that system (26) admits a comparison principle, see Ishii and Koike (1991), which we use for the proof of convergence.

Proposition 3. The following is true:

(A1) Let $u=\left(u_{q}\right), v=\left(v_{q}\right) \in \mathbb{R}^{n}$. Suppose that $u_{q_{*}}-v_{q_{*}}=\max _{q}\left\{u_{q}-v_{q}\right\} \geqslant 0$. Then

$$
F_{q_{*}}(\tau, S, u, c, p, X)-F_{q_{*}}(\tau, S, v, c, p, X) \geqslant \kappa\left(u_{q_{*}}-v_{q_{*}}\right) .
$$

(A2) There exists $\beta>1$ and a continuous function $\omega: \mathbb{R}_{+} \rightarrow \mathbb{R}_{+}, \omega(0)=0$, such that for any $X, X^{\prime} \in \mathbb{R}$ satisfying (in the sense of quadratic forms)

$$
-3 \beta\left(\begin{array}{cc}
1 & 0 \\
0 & 1
\end{array}\right) \leqslant\left(\begin{array}{cc}
X & 0 \\
0 & X^{\prime}
\end{array}\right) \leqslant 3 \beta\left(\begin{array}{cc}
1 & -1 \\
-1 & 1
\end{array}\right)
$$

we have

$$
\begin{aligned}
F_{q}\left(\tau, S, u, \beta\left(\tau^{\prime}-\tau\right), \beta\left(S^{\prime}-S\right),-X\right)-F_{q}\left(\tau^{\prime}, S^{\prime}, u, \beta\left(\tau^{\prime}-\tau\right), \beta\left(S^{\prime}-S\right), X^{\prime}\right) \\
\leqslant \omega\left(\beta\left(\tau^{\prime}-\tau\right)^{2}+\beta\left(S^{\prime}-S\right)^{2}+\beta^{-1}\right)
\end{aligned}
$$

for all $q$. 


\subsection{Finite difference scheme}

We provide a finite difference scheme $F^{\varepsilon, h}=\left(F_{q}^{\varepsilon, h}\right):[0, \bar{T}] \times \mathbb{R} \times \mathbb{R}^{m} \times C([0, \bar{T}] \times \mathbb{R}) \rightarrow \mathbb{R}^{m}$ by the formula $\left(r=\left(r_{q}\right) \in \mathbb{R}^{m}, \varphi \in C([0, \bar{T}] \times \mathbb{R})\right.$, where $\left.m=\bar{q}-\underline{q}+1\right)$

$$
\begin{aligned}
& F_{q}^{\varepsilon, h}(\tau, S, r, \varphi):=\max \left\{\frac{r_{q}-\varphi(\tau-\varepsilon, S)}{\varepsilon}-\right. \frac{1}{2} \sigma^{2} \frac{\varphi(\tau, S+h)-2 r_{q}+\varphi(\tau, S-h)}{h^{2}}+\tilde{A}(\bar{T}-\tau, S) q-\phi(q)^{2} \\
&-\tilde{A}^{+}(\bar{T}-\tau, S)\left(\frac{\varphi(\tau, S+h)-r_{q}}{h}\right)-\tilde{A}^{-}(\bar{T}-\tau, S)\left(\frac{r_{q}-\varphi(\tau, S-h)}{h}\right) \\
&+\mathbb{1}_{q<\bar{q}} \lambda^{-} \max _{\ell \in\{0,1\}}\left[\frac{\Delta}{2} \ell+(1+\kappa) r_{q}-r_{q+\ell}\right] \\
&+\mathbb{1}_{q>\underline{q}} \lambda^{+} \max _{\ell \in\{0,1\}}\left[\frac{\Delta}{2} \ell+(1+\kappa) r_{q}-r_{q-\ell}\right] \\
&\left.\max _{\xi \in\left\{-\mathbb{1}_{q>\underline{q}}, 0, \mathbb{1}_{q<\bar{q}}\right\}}\left\{-\left(\frac{\Delta}{2}+\varepsilon\right)|\xi|+(1+\kappa) r_{q}-r_{q+\xi}\right\}\right\},
\end{aligned}
$$

where $\tilde{A}^{+}=\max \{0, \tilde{A}\}, \tilde{A}^{-}=\min \{0, \tilde{A}\}$ on $[0, \bar{T}] \times \mathbb{R}$.

Let

$$
\mathbb{S}_{h}:=\left\{S_{k}:=h k: k \in \mathbb{Z}\right\}, \quad \mathbb{T}_{\varepsilon}=\left\{\tau_{j}:=\varepsilon j: 0 \leqslant j \leqslant M\right\},
$$

where $\varepsilon>0$ is chosen to that $M:=\frac{\bar{T}}{\varepsilon} \in \mathbb{Z}_{+}$. We show that solutions $u^{\varepsilon, h}=\left(u_{q}^{\varepsilon, h}\right), u_{q}^{\varepsilon, h}$ : $\mathbb{T}_{\varepsilon} \times \mathbb{S}_{h} \rightarrow \mathbb{R}(\varepsilon, h>0)$ of the discrete problem

$$
\begin{gathered}
F_{q}^{\varepsilon, h}\left(\tau_{j}, S_{k}, u^{\varepsilon, h}\left(\tau_{j}, S_{k}\right), u_{q}^{\varepsilon, h}\right)=0, \quad j, k \in \mathbb{Z}, \\
u_{q}^{\varepsilon, h}\left(0, S_{k}\right)=-\left(\frac{\Delta}{2}+\varepsilon\right)|q|+\alpha(q)^{2}, \quad \underline{q} \leqslant q \leqslant \bar{q}, \quad k \in \mathbb{Z},
\end{gathered}
$$

can be used to approximate the solution $u=\left(u_{q}\right)$ of $(26)$.

Proposition 4. The following are true:

(C1) For all bounded functions $u=\left(u_{q}\right), v=\left(v_{q}\right)$ with $u_{q}, v_{q} \in C([0, \bar{T}] \times \mathbb{R})$ and $u_{q} \leqslant v_{q}$ on $[0, \bar{T}] \times \mathbb{R}$, and any $r, s \in \mathbb{R}^{n}$ such that $(\gamma=) r_{q^{*}}-s_{q^{*}}=\max _{q}\left\{r_{q}-s_{q}\right\} \geqslant 0$ we have

$$
F_{q_{*}}^{\varepsilon, h}\left(\tau, S, r, u_{q_{*}}+\gamma\right)-F_{q_{*}}^{\varepsilon, h}\left(\tau, S, s, v_{q_{*}}\right) \geqslant \kappa \gamma .
$$

(C2) For any bounded function $\varphi \in C([0, \bar{T}] \times \mathbb{R})$, fixed $(\tau, S) \in[0, \bar{T}] \times \mathbb{R}$ and $r=\left(r_{q}\right) \in \mathbb{R}^{n}$, the functions

$$
S \mapsto F_{q}^{\varepsilon, h}(\tau, S, r, \varphi), \quad \underline{q} \leqslant q \leqslant \bar{q},
$$

are bounded and continuous, and the functions

$$
r \mapsto F_{q}^{\varepsilon, h}(\tau, S, r, \varphi), \quad \underline{q} \leqslant q \leqslant \bar{q},
$$

are uniformly continuous for $r$ in a bounded set, uniformly in $S \in \mathbb{R}^{n}$.

(C3) For every $\psi=\left(\psi_{q}\right), \psi_{q} \in C\left([0, \bar{T}] \times \mathbb{R}^{n}\right) \cap C^{1,1}((0, \bar{T}) \times \mathbb{R}),(\tau, S) \in[0, \bar{T}] \times \mathbb{R}$ and $\delta>0$ 
there exist $h, \varepsilon>0$ such that

$$
\left|F_{q}^{\varepsilon, h}\left(\tau, S, \psi_{q}(\tau, S), \psi\right)-F_{q}\left(\tau, S, \psi, \partial_{S} \psi, \partial_{S S} \psi\right)\right|<\delta, \quad \underline{q} \leqslant q \leqslant \bar{q} .
$$

(C4) For any $\varepsilon, h>0$ there exists a bounded solution $u^{\varepsilon, h}=\left(u_{q}^{\varepsilon, h}\right), u_{q}^{\varepsilon, h}: \mathbb{T}_{\varepsilon} \times \mathbb{S}_{h} \rightarrow \mathbb{R}$ of the discrete problem (27).

Armed with these results, we define the candidate super- and sub-solutions of (26) as

$$
\begin{aligned}
& \underline{u}_{q}(\tau, S)=\liminf _{\varepsilon, h \rightarrow+0,\left(\tau^{\prime}, S^{\prime}\right) \rightarrow(\tau, S)} u_{q}^{\varepsilon, h}\left(\tau^{\prime}, S^{\prime}\right), \\
& \bar{u}_{q}(\tau, S)=\limsup _{\varepsilon, h \rightarrow+0,\left(\tau^{\prime}, S^{\prime}\right) \rightarrow(\tau, S)} u_{q}^{\varepsilon, h}\left(\tau^{\prime}, S^{\prime}\right),
\end{aligned}
$$

for $(\tau, S) \in[0, \bar{T}] \times \mathbb{R}, \underline{q} \leqslant q \leqslant \bar{q}$.

Propositions 3 and 4 allow us to apply the argument of Proposition 3.3 of Briani et al. (2012): namely, $\underline{u}=\left(\underline{u}_{q}\right)$ and $\bar{u}=\left(\bar{u}_{q}\right)$ are indeed, respectively, supersolution and subsolution of (26), and hence by the comparison principle (cf. remark before Proposition 3 ) $\bar{u}_{q} \leqslant \underline{u}_{q}$ on $[0, \bar{T}] \times \mathbb{R}$. The opposite inequality is obviously true, and hence $u:=\bar{u}=\underline{u}$ is the viscosity solution of (26). The constructed solution is bounded, since a comparison principle holds and the fact that (24)-(25) admits constant sub- and super- solutions, see Proposition 6.1 in the Appendix of Briani et al. (2012).

\section{Conclusions}

In this paper we show how an algorithmic trader learns from market-wide price dynamics to trade around directional predictions of the price of an asset or collection of assets. The profits of the strategy come from both capital appreciation of inventories and earning the spread from round-trip trades. When the quality of the information is impaired by the volatility of prices, the strategy relies less on betting on the direction of the asset and relies more on two-sided quotes to earn the spread. In extreme cases, where the trader is uniformed or the quality of the information she gathers is too poor, the strategy behaves like that of a market maker who is willing to bear little inventory risk.

We also show how learning from other assets that co-move increases the profits of the trading strategies. This increase in profits is noticeable when the trader takes positions in an asset which exhibits high volatility but improves the quality of the information she gathers by learning from the dynamics of other assets that co-move. We also show how the trader's strategy is modified to account for adverse selection costs.

Finally, we show that the numerical scheme does indeed converge to the unique viscosity solution of the QVI that arises from the dynamic programming principle. 


\section{Appendix A. Proofs}

Proof (Proposition 1). We conduct our proof in two steps.

1. First, we show that conditional expectations $a_{i}\left(t, y_{1}, \ldots, y_{d}\right)$ are indeed given by formula (6). By definition

$$
a_{i}\left(t, y_{1}, \ldots, y_{n}\right)=\int_{-\infty}^{\infty} x_{i} d F^{i}\left(x_{i} \mid \mathbf{S}_{t}=\mathbf{y}\right)
$$

where $F^{i}\left(x \mid \mathbf{S}_{t}=\mathbf{y}\right)$ is the conditional cumulative distribution function (cdf) of $D^{i}$. We have

$$
F^{i}\left(x_{i} \mid \mathbf{S}_{t}=\mathbf{y}\right)=F\left(+\infty, \ldots, x_{i}, \ldots,+\infty \mid \mathbf{S}_{t}=\mathbf{y}\right),
$$

where $F\left(\mathbf{x} \mid \mathbf{S}_{t}=\mathbf{y}\right)$ is the joint conditional cdf of the random variables $\mathbf{D}$. Using Bayes' formula

$$
F\left(\mathbf{x} \mid \mathbf{S}_{t}=\mathbf{y}\right)=\frac{\int_{-\infty}^{x_{1}} \cdots \int_{-\infty}^{x_{n}} p_{\mathbf{S}_{t}}(\mathbf{y} \mid \mathbf{D}=\mathbf{z}) d F(\mathbf{z})}{\int_{\mathbb{R}^{n}} p_{\mathbf{S}_{t}}(\mathbf{y} \mid \mathbf{D}=\mathbf{z}) d F(\mathbf{z})},
$$

where $p_{\mathbf{S}_{t}}(\mathbf{y} \mid \mathbf{D}=\mathbf{z})$ is the joint conditional probability density function (pdf) of $\mathbf{S}_{t}$. Conditional on $\mathbf{D}=\mathbf{z}, S_{t}^{i}=\sigma_{i} \beta_{t T}^{i}+\frac{t}{T} z_{i}$ are independent Gaussian random variables with mean $\frac{t}{T} z_{i}$ and variance $\sigma_{i}^{2} \frac{t}{T}(T-t)$. This provides an explicit formula for $p_{\mathbf{S}_{t}}(\mathbf{y} \mid \mathbf{D}=\mathbf{z})$, and as a result we obtain (6).

2. Next, we show that $\mathbf{S}_{t}$ satisfies (4). We fix some $i \in\{1,2 \ldots, n\}$, it suffices to show that

$$
W_{\tau}^{i}=\frac{1}{\sigma_{i}}\left(S_{t}^{i}-S_{0}^{i}+\int_{0}^{\tau} \frac{S_{u}^{i}-S_{0}^{i}}{T-u} d u-\int_{0}^{\tau} \frac{a_{i}\left(u, \mathbf{S}_{u}\right)}{T-u} d u\right)
$$

is an $\mathcal{F}_{\tau}$-Brownian motion. By definition, $W_{\tau}^{i}$ is $\mathcal{F}_{\tau}$-adapted. It follows from $(2)$ that $d\left[W^{i}, W^{i}\right]_{\tau}=$ $d \tau$. Therefore, by Lévy's theorem, it remains to verify that $W_{\tau}^{i}$ is a martingale. We have for $t \leqslant \tau$

$$
\begin{aligned}
\sigma_{i} \mathbb{E}\left[W_{\tau}^{i} \mid \mathcal{F}_{t}\right]= & \mathbb{E}\left[S_{\tau}^{i}-S_{0}^{i} \mid \mathcal{F}_{t}\right]+\int_{0}^{t} \frac{S_{u}^{i}-S_{0}^{i}}{T-u} d u-\int_{0}^{t} \frac{a_{i}\left(u, \mathbf{S}_{u}\right)}{T-u} d u \\
& +\int_{t}^{\tau} \frac{\mathbb{E}\left[S_{u}^{i}-S_{0}^{i} \mid \mathcal{F}_{t}\right]}{T-u} d u-\int_{t}^{\tau} \frac{\mathbb{E}\left[a_{i}\left(u, \mathbf{S}_{u}\right) \mid \mathcal{F}_{t}\right]}{T-u} d u \\
:= & I_{1}+I_{2}-I_{3}+I_{4}-I_{5} .
\end{aligned}
$$

Using identity $\mathbb{E}\left[\beta_{u T} \mid \mathcal{F}_{t}\right]=\frac{T-u}{T-t} \mathbb{E}\left[\beta_{t T} \mid \mathcal{F}_{t}\right]$ (since $\beta_{t T}$ is a standard Brownian bridge), we obtain:

$$
\begin{aligned}
I_{1} & =\sigma_{i} \mathbb{E}\left[\beta_{\tau T}^{i} \mid \mathcal{F}_{t}\right]+\frac{\tau}{T} \mathbb{E}\left[D^{i} \mid \mathcal{F}_{t}\right]=\sigma_{1} \frac{T-\tau}{T-t} \mathbb{E}\left[\beta_{t T} \mid \mathcal{F}_{t}\right]+\frac{\tau}{T} a_{i}\left(t, \mathbf{S}_{t}\right), \\
I_{4} & =a_{i}(t, \mathbf{S}) \int_{t}^{\tau} \frac{1}{T-s} d s \\
I_{5} & =\int_{t}^{\tau} \frac{1}{T-u}\left(\sigma_{i} \frac{T-u}{T-t} \mathbb{E}\left[\beta_{t T} \mid \mathcal{F}_{t}\right]+\frac{u}{T} a_{i}\left(t, \mathbf{S}_{t}\right)\right) d u
\end{aligned}
$$


where the second and third equalities follow from (2) and the law of iterated expectations. Furthermore, re-arranging (A.1) yields

$$
I_{2}-I_{3}=\sigma_{i} W_{t}^{i}-\left(S_{t}^{i}-S_{0}^{i}\right) .
$$

Therefore, identity (A.2) can be re-written as

$$
\mathbb{E}\left[W_{u}^{i} \mid \mathcal{F}_{t}\right]=\frac{1}{\sigma_{i}}\left(\sigma_{i} \mathbb{E}\left[\beta_{t T} \mid \mathcal{F}_{t}\right]+\sigma_{i} W_{t}^{i}-S_{t}^{i}+S_{0}^{i}+\frac{t}{T} a_{i}(t, \mathbf{S})\right) .
$$

By definition,

$$
S_{t}^{i}-S_{0}^{i}=\mathbb{E}\left[S_{t}^{i}-S_{0}^{i} \mid \mathcal{F}_{t}\right]=\sigma_{i} \mathbb{E}\left[\beta_{t T} \mid \mathcal{F}_{t}\right]+\frac{t}{T} a_{i}(t, \mathbf{S}),
$$

and hence we see from the previous formula that $W_{t}^{i}$ satisfies the martingale condition.

Finally, it follows from (2) that $d\left[W^{i}, W^{j}\right]_{\tau}=0$ if $i \neq j$. Therefore, by Lévy's theorem, independent $\mathcal{F}_{t}$-Brownian motions.

Proof (Proposition 2). A straightforward generalization of Øksendal and Sulem (2008), Theorem 10.8, to a vector-valued stochastic-impulse controlled process implies that the solution $H$ of the optimization problem (14) is given by a viscosity solution to the following QVI, for all $0<t<\bar{T}, X \in \mathbb{R}, \mathbf{S} \in \mathbb{R}, q^{i}=\left\{\underline{q}^{i}, \ldots, \bar{q}^{i}\right\}:$

$$
\begin{aligned}
& 0=\max \left\{\partial_{t} H\right.+\sum_{i=1}^{n}\left\{\frac{1}{2} \sigma_{i}^{2} \partial_{S^{i} S^{i}} H+A_{i}(t, \mathbf{S}) \partial_{S^{i}} H\right\}-\sum_{i=1}^{k} \phi^{i}\left(q^{i}\right)^{2} \\
&+\sum_{i=1}^{k} \mathbb{1}_{q^{i}<\bar{q}^{i}} \lambda^{i-} \max _{\ell \in\{0,1\}}\left[H\left(t, X-\ell\left(S^{i}-\frac{\Delta^{i}}{2}\right), \mathbf{S}, \mathbf{q}+\boldsymbol{\delta}^{i} \ell\right)-H\right] \\
&+\sum_{i=1}^{k} \mathbb{1}_{q^{i}>\underline{q}^{i}} \lambda^{i+} \max _{\ell \in\{0,1\}}\left[H\left(t, X+\ell\left(S^{i}+\frac{\Delta^{i}}{2}\right), \mathbf{S}, \mathbf{q}-\boldsymbol{\delta}^{i} \ell\right)-H\right] ; \\
&\left.\max _{\boldsymbol{\xi} \in \mathcal{D}(\mathbf{q})}\left\{-\sum_{i=1}^{k}\left(H\left(t, X-\xi^{i}\left(S+\frac{\Delta^{i}}{2} \xi^{i}\right)-\varepsilon^{i}\left|\xi^{i}\right|, \mathbf{S}, \mathbf{q}+\boldsymbol{\xi}\right)-H\right)\right\}\right\},
\end{aligned}
$$

subject to the terminal condition

$$
H(\bar{T}, X, \mathbf{S}, \mathbf{q})=X+\sum_{i=1}^{k} \psi^{i}\left(q^{i}, S^{i}\right), \quad \underline{q} \leqslant q \leqslant \bar{q} .
$$

Substituting the ansatz (15) into the above DPE leads to the stated QVI for $g$. In Section 6, we prove that (A.3) has at least one solution $g$. By the comparison principle (also established in Section 6), this solution is unique.

Proof (Proposition 3). To simplify notation, we assume without loss of generality that $\lambda^{+}=\lambda_{-}=1$. We write

$$
F_{q}(\tau, S, u, c, p, X)=\max \left\{L_{q}(\tau, S, u, c, p, X), M_{q}(\tau, S, u, c, p, X)\right\},
$$


where

$$
\begin{aligned}
L_{q}(\tau, S, u, c, p, X)=c_{q}- & \frac{1}{2} \sigma^{2} X_{q}+\tilde{A}(\bar{T}-\tau, S)\left(q-p_{q}\right)-\phi q^{2} \\
& +\mathbb{1}_{q<\bar{q}} \lambda^{-} \max _{\ell \in\{0,1\}}\left[\frac{\Delta}{2} \ell+(1+\kappa) u_{q}-u_{q+\ell}\right] \\
& +\mathbb{1}_{q>\underline{q}} \lambda^{+} \max _{\ell \in\{0,1\}}\left[\frac{\Delta}{2} \ell+(1+\kappa) u_{q}-u_{q-\ell}\right],
\end{aligned}
$$

and

$$
M_{q}(\tau, S, u, c, p, X)=\max _{\xi \in\left\{-\mathbb{1}_{q>\underline{q}}, 0, \mathbb{1}_{q<\bar{q}}\right\}}\left\{-\left(\frac{\Delta}{2}+\varepsilon\right)|\xi|+(1+\kappa) u_{q}-u_{q+\xi}\right\},
$$

for $\underline{q} \leqslant q \leqslant \bar{q}$.

(A1) The required inequality can be stated as follows:

$\max \left\{L_{q}(\tau, S, u, c, p, X), M_{q}(\tau, S, u, c, p, X)\right\}-\max \left\{L_{q}(\tau, S, v, c, p, X), M_{q}(\tau, S, v, c, p, X)\right\} \geqslant 0$.

Depending on where the maximum is attained, the latter is equivalent to one of the following inequalities:

1. $L_{q^{*}}(\tau, S, u, c, p, X)-L_{q^{*}}(\tau, S, v, c, p, X) \geqslant 0$,

2. $M_{q^{*}}(\tau, S, u, c, p, X)-L_{q^{*}}(\tau, S, v, c, p, X) \geqslant 0$,

3. $L_{q^{*}}(\tau, S, u, c, p, X)-M_{q^{*}}(\tau, S, v, c, p, X) \geqslant 0$,

4. $M_{q^{*}}(\tau, S, u, c, p, X)-M_{q^{*}}(\tau, S, v, c, p, X) \geqslant 0$.

In fact, it is easy to see that the proof of 1) and 4) would suffice.

Let us prove 1) i.e. that in case $\underline{q}<q^{*}<\bar{q}$

$$
\begin{aligned}
& \max \left\{0, \frac{\Delta}{2}+u_{q^{*}}-u_{q^{*}-1}\right\}-\max \left\{0, \frac{\Delta}{2}+v_{q^{*}}-v_{q^{*}-1}\right\} \geqslant 0, \\
& \max \left\{0, \frac{\Delta}{2}+u_{q^{*}}-u_{q^{*}+1}\right\}-\max \left\{0, \frac{\Delta}{2}+v_{q^{*}}-v_{q^{*}+1}\right\} \geqslant 0,
\end{aligned}
$$

(if $q=\underline{q}$ or $q=\bar{q}$ one of the above inequalities is void). We prove the last inequality: it can be violated only if $\frac{\Delta}{2}+u_{q^{*}}<u_{q^{*}+1}, \frac{\Delta}{2}+v_{q^{*}}>v_{q^{*}+1}$. Then $-\frac{\Delta}{2}-v_{q^{*}}<-v_{q^{*}+1}$. Adding up the latter and $\frac{\Delta}{2}+u_{q^{*}}<u_{q^{*}+1}$ we obtain $u_{q^{*}}-v_{q^{*}}<u_{q^{*}+1}-v_{q^{*}+1}$, which contradicts out assumption that $u_{q^{*}}-v_{q^{*}}=\max _{q}\left(u_{q}-v_{q}\right)$.

Now, let us prove 4), i.e. that

$$
\begin{gathered}
\max \left\{0,-\frac{\Delta}{2}-\varepsilon+u_{q^{*}}-u_{q^{*}+1},-\frac{\Delta}{2}-\varepsilon+u_{q^{*}}-u_{q^{*}-1}\right\} \\
-\max \left\{0,-\frac{\Delta}{2}-\varepsilon+v_{q^{*}}-v_{q^{*}+1},-\frac{\Delta}{2}-\varepsilon+v_{q^{*}}-v_{q^{*}-1}\right\} \geqslant 0 .
\end{gathered}
$$

The proof is straightforward: for instance, suppose that the first maximum is attained at the second argument, while the second maximum is attained at the third argument; then the inequality that we need to prove becomes

$$
-\frac{\Delta}{2}-\varepsilon+u_{q^{*}}-u_{q^{*}+1}-\left(-\frac{\Delta}{2}-\varepsilon+v_{q^{*}}-v_{q^{*}-1}\right) \geqslant 0
$$


i.e. $u_{q^{*}}-u_{q^{*}+1}-\left(v_{q^{*}}-v_{q^{*}-1}\right) \geqslant 0$. Noting that $u_{q^{*}}-u_{q^{*}+1} \geqslant u_{q^{*}}-u_{q^{*}-1}$, and using our assumption $u_{q^{*}}-v_{q^{*}}=\max _{q}\left(u_{q}-v_{q}\right)$, we obtain the required inequality.

(A2) The condition on $X, X^{\prime} \in \mathbb{R}$, re-written in coordinates, is as follows:

$$
-3 \beta\left(x^{2}+\left(x^{\prime}\right)^{2}\right) \leqslant X x^{2}+X^{\prime}\left(x^{\prime}\right)^{2} \leqslant 3 \beta\left(x-x^{\prime}\right)^{2} \quad \text { for all } \quad\left(x, x^{\prime}\right) \in \mathbb{R}^{2} .
$$

We need to prove

$$
\begin{array}{r}
\max \left\{L_{q}\left(\tau, S, u, \beta\left(\tau^{\prime}-\tau\right), \beta\left(S^{\prime}-S\right),-X\right), M_{q}\left(\tau, S, u, \beta\left(\tau^{\prime}-\tau\right), \beta\left(S^{\prime}-S\right),-X\right)\right\} \\
-\max \left\{L_{q}\left(\tau^{\prime}, S^{\prime}, u, \beta\left(\tau^{\prime}-\tau\right), \beta\left(S^{\prime}-S\right), X^{\prime}\right), M_{q}\left(\tau^{\prime}, S^{\prime}, u, \beta\left(\tau^{\prime}-\tau\right), \beta\left(S^{\prime}-S\right), X^{\prime}\right)\right\} \\
\leqslant \omega\left(\beta\left(\tau^{\prime}-\tau\right)^{2}+\beta\left(S^{\prime}-S\right)^{2}+\beta^{-1}\right) .
\end{array}
$$

Similarly to the proof of (A1), it suffices to show that

$$
\begin{aligned}
& \begin{array}{c}
L_{q}\left(\tau, S, u, \beta\left(\tau^{\prime}-\tau\right), \beta\left(S^{\prime}-S\right),-X\right)-L_{q}\left(\tau^{\prime}, S^{\prime}, u, \beta\left(\tau^{\prime}-\tau\right), \beta\left(S^{\prime}-S\right), X^{\prime}\right) \\
\leqslant \omega\left(\beta\left(\tau^{\prime}-\tau\right)^{2}+\beta\left(S^{\prime}-S\right)^{2}+\beta^{-1}\right), \\
M_{q}\left(\tau, S, u, \beta\left(\tau^{\prime}-\tau\right), \beta\left(S^{\prime}-S\right),-X\right)-M_{q}\left(\tau^{\prime}, S^{\prime}, u, \beta\left(\tau^{\prime}-\tau\right), \beta\left(S^{\prime}-S\right), X^{\prime}\right) \\
\leqslant \omega\left(\beta\left(\tau^{\prime}-\tau\right)^{2}+\beta\left(S^{\prime}-S\right)^{2}+\beta^{-1}\right) .
\end{array}
\end{aligned}
$$

The second inequality is trivially true for any continuous $\omega: \mathbb{R}_{+} \rightarrow \mathbb{R}_{+}, \omega(0)=0$ since its left-hand is identically zero. Let us prove the first inequality. We have

$$
\begin{aligned}
& L_{q}\left(\tau, S, u, \beta\left(\tau^{\prime}-\tau\right), \beta\left(S^{\prime}-S\right),-X\right)-L_{q}\left(\tau^{\prime}, S^{\prime}, u, \beta\left(\tau^{\prime}-\tau\right), \beta\left(S^{\prime}-S\right), X^{\prime}\right) \\
& =\left(\tilde{A}(\bar{T}-\tau, S)-\tilde{A}\left(\bar{T}-\tau^{\prime}, S^{\prime}\right)\right) q+\tilde{A}(\bar{T}-\tau, S) \beta\left(S^{\prime}-S\right)-\tilde{A}\left(\bar{T}-\tau^{\prime}, S^{\prime}\right) \beta\left(S^{\prime}-S\right)+\frac{1}{2} \sigma^{2}\left(X+X^{\prime}\right) .
\end{aligned}
$$

The last term is negative, according to our assumption on $X, X^{\prime}$, cf. (A.7) with $x=x^{\prime}=1$. The rest now follows from the fact that $\tilde{A}(\bar{T}-\tau, S)$ is globally Lipschitz in $(\tau, S)$, cf. Section 2.

Proof (Proposition 4). The verification of conditions (C2), (C3) and (C4) is immediate. Let us show that condition $(\mathrm{C} 1)$ is satisfied as well. Denote

$$
F_{q}^{\varepsilon, h}(\tau, S, r, \varphi)=\max \left\{L_{q}^{\varepsilon, h}(\tau, S, r, \varphi), M_{q}^{\varepsilon, h}(\tau, S, r, \varphi)\right\}
$$

Similarly to the proof of Proposition 3, it suffices to prove the following two inequalities:

$$
\begin{gathered}
L_{q_{*}}^{\varepsilon, h}\left(\tau, S, r, u_{q_{*}}+\gamma\right)-L_{q_{*}}^{\varepsilon, h}\left(\tau, S, s, v_{q_{*}}\right) \geqslant \kappa \gamma, \\
M_{q_{*}}^{\varepsilon, h}\left(\tau, S, r, u_{q_{*}}+\gamma\right)-M_{q_{*}}^{\varepsilon, h}\left(\tau, S, s, v_{q_{*}}\right) \geqslant \kappa \gamma .
\end{gathered}
$$

The proof of the second inequality essentially repeats the proof of inequality 4) in the proof of Proposition 3. For the proof of the first inequality, let us write $L_{q_{*}}^{\varepsilon, h}\left(\tau, S, r, u_{q_{*}}+\gamma\right)-$ 


$$
\begin{aligned}
& L_{q_{*}}^{\varepsilon, h}\left(\tau, S, s, v_{q_{*}}\right)=I_{1}+I_{2}, \text { where } \\
& I_{1}= \frac{r_{q_{*}}-u(\tau-\varepsilon, S)-\gamma}{\varepsilon}-\frac{s_{q}-v(\tau-\varepsilon, S)}{\varepsilon} \\
&-\frac{1}{2} \sigma^{2} \frac{u(\tau, S+h)-2 r_{q_{*}}+u(\tau, S-h)+2 \gamma}{h^{2}}+\frac{1}{2} \sigma^{2} \frac{v(\tau, S+h)-2 s_{q_{*}}+v(\tau, S-h)}{h^{2}} \\
&-\tilde{A}^{+}(\bar{T}-\tau, S)\left(\frac{u(\tau, S+h)+\gamma-r_{q_{*}}}{h}-\frac{v(\tau, S+h)-s_{q_{*}}}{h}\right) \\
&-\tilde{A}^{-}(\bar{T}-\tau, S)\left(\frac{r_{q_{*}}-u(\tau, S-h)-\gamma}{h}-\frac{s_{q_{*}}-v(\tau, S-h)}{h}\right)
\end{aligned}
$$

and

$$
\begin{aligned}
I_{2}= & \mathbb{1}_{q_{*}<\bar{q}} \lambda^{-} \max _{\ell \in\{0,1\}}\left[\frac{\Delta}{2} \ell+(1+\kappa) r_{q_{*}}-r_{q_{*}+\ell}\right]+\mathbb{1}_{q_{*}>\underline{q}} \lambda^{+} \max _{\ell \in\{0,1\}}\left[\frac{\Delta}{2} \ell+(1+\kappa) r_{q_{*}}-r_{q_{*}-\ell}\right] \\
& -\mathbb{1}_{q_{*}<\bar{q}} \lambda^{-} \max _{\ell \in\{0,1\}}\left[\frac{\Delta}{2} \ell+(1+\kappa) s_{q_{*}}-s_{q_{*}+\ell}\right]-\mathbb{1}_{q_{*}>\underline{q}} \lambda^{+} \max _{\ell \in\{0,1\}}\left[\frac{\Delta}{2} \ell+(1+\kappa) s_{q_{*}}-s_{q_{*}-\ell}\right] .
\end{aligned}
$$

The proof that $I_{2} \geqslant \kappa \gamma$ is similar to the proof of inequality 1) in the proof of Proposition 3 . It remains to show that $I_{1} \geqslant 0$. The latter is immediate from our assumptions $u \leqslant v$ and $\gamma=r_{q^{*}}-s_{q^{*}}=\max _{q}\left\{r_{q}-s_{q}\right\} \geqslant 0$.

\section{References}

Almgren, R. (2003). Optimal execution with nonlinear impact functions and trading-enhanced risk. Applied Mathematical Finance 10(1), 1-18.

Avellaneda, M. and S. Stoikov (2008, November). High-frequency trading in a limit order book. Quantitative Finance 8, 217-224.

Barles, G. and P. Soganidis (1991). Convergence of approximation schemes for fully nonlinear second order equations. Asymptotic Analysis $\underline{4}$, 271-283.

Baudoin, F. (2002). Conditional SDE and applications in finance. Stochastic Processes and their Applications 100, 109-145.

Baudoin, F. (2003). Modelling anticipation on a financial market. Paris Princeton Lecture Notes on Mathematical Finance LNM 1814.

Baudoin, F. and L. Nguyen-Ngo (2004). The financial value of weak information. Finance and $\underline{\text { Stochastics }} \underline{8}, 415-435$.

Bayraktar, E. and M. Ludkovski (2011). Optimal trade execution in illiquid markets. Mathematical Finance 21(4), 681-701. 
Bayraktar, E. and M. Ludkovski (2014). Liquidation in limit order books with controlled intensity. Mathematical Finance 24(4), 627-650.

Becherer, D. and M. Schweizer (2005). Classical solutions to reaction-diffusion systems for hedging problems with interacting Itô and point processes. Annals of Applied Probability 15, $1111-1144$.

Bechler, K. and M. Ludkovski (2014). Optimal execution with dynamic order flow imbalance. arXiv preprint arXiv:1409.2618.

Briani, A., F. Camini, and H. Zidani (2012). Approximation schemes for monotone systems of nonlinear second order parabolic equations: convergence result and error estimate. Differential Equations and Applications 4, 297-317.

Brody, D., L. Hughston, and A. Macrina (2008). Information-based asset pricing. International Journal of Theoretical and Applied Finance 11, 107-142.

Brody, D. C., M. H. Davis, R. L. Friedman, and L. P. Hughston (2009). Informed traders. In Proceedings of the Royal Society of London A: Mathematical, Physical and Engineering Sciences, Volume 465, pp. 1103-1122. The Royal Society.

Cartea, Á., R. Donnelly, and S. Jaimungal (2013). Algorithmic trading with model uncertainty. Social Science Research Network Working Paper Series.

Cartea, Á., R. F. Donnelly, and S. Jaimungal (2015). Enhancing trading strategies with order book signals. Available at SSRN 2668277.

Cartea, Á. and S. Jaimungal (2013). Modeling asset prices for algorithmic and high frequency trading. Applied Mathematical Finance 20(6), 512-547.

Cartea, Á. and S. Jaimungal (2014). Incorporating order-flow into optimal execution. Available at SSRN 2557457.

Cartea, Á. and S. Jaimungal (2015a). Optimal execution with limit and market orders. Quantitative Finance 15(8), 1279-1291.

Cartea, Á. and S. Jaimungal (2015b). Risk metrics and fine tuning of high-frequency trading strategies. Mathematical Finance 25(3), 576-611.

Cartea, Á., S. Jaimungal, and J. Penalva (2015). Algorithmic and High-Frequency Trading. Cambridge University Press.

Cartea, Á., S. Jaimungal, and J. Ricci (2014). Buy low, sell high: A high frequency trading perspective. SIAM Journal on Financial Mathematics 5 (1), 415-444. 
Cartea, Á., S. Jaimungal, and J. Ricci (2015). Trading strategies within the edges of noarbitrage. Social Science Research Network Working Paper Series.

Gatheral, J., A. Schied, and A. Slynko (2012). Transient linera price impact and Fredholm integral equations. Mathematical Finance 22(3), 445-474.

Guéant, O. and C.-A. Lehalle (2015). General intensity shapes in optimal liquidation. Mathematical Finance 25(3), 457-495.

Guéant, O., C.-A. Lehalle, and J. Fernandez-Tapia (2012). Dealing with the inventory risk: a solution to the market making problem. Mathematics and Financial Economics, 1-31.

Guilbaud, F. and H. Pham (2013). Optimal high-frequency trading with limit and market orders. Quantitative Finance 13(1), 79-94.

Ho, T. and H. R. Stoll (1981). Optimal dealer pricing under transactions and return uncertainty. Journal of Financial Economics 9 , 47-73.

Ishii, H. and S. Koike (1991). Viscosity solutions for monotone systems of second order elliptic pdes. Communications in Partial Differential Equations 16, 1095-1128.

Kharroubi, I. and H. Pham (2010). Optimal portfolio liquidation with execution cost and risk. SIAM Journal on Financial Mathematics 1, 897-931.

Lipton, A., U. Pesavento, and M. G. Sotiropoulos (2013). Trade arrival dynamics and quote imbalance in a limit order book. arXiv:1312.0514.

Macrina, A. (2014). Heat kernel models for asset pricing. International Journal of Theoretical and Applied Finance 17(07), 1450048.

Obizhaeva, A. A. and J. Wang (2013). Optimal trading strategy and supply/demand dynamics. Journal of Financial Markets 16(1), 1-32.

Øksendal, B. and A. Sulem (2008). Applied stochastic control of jump diffusions, 3rd ed. Springer.

Rogers, L. C. G. and D. Williams (1987). Diffusions, Markov processes, and martingales, Volume 2. John Wiley \& Sons Inc.

Schied, A. (2013). Robust strategies for optimal order execution in the Almgren-Chriss framework. Applied Mathematical Finance 20(3), 264-286. 